\title{
A fixed point formula of Lefschetz type in Arakelov geometry III: representations of Chevalley schemes and heights of flag varieties
}

\author{
Christian Kaiser \\ Kai Köhler
}

November 14, 2018

\begin{abstract}
We give a new proof of the Jantzen sum formula for integral representations of Chevalley schemes over Spec $\mathbf{Z}$. This is done by applying the fixed point formula of Lefschetz type in Arakelov geometry to generalized flag varieties. Our proof involves the computation of the equivariant Ray-Singer torsion for all equivariant bundles over complex homogeneous spaces. Furthermore, we find several explicit formulae for the global height of any generalized flag variety.
\end{abstract}

2000 Mathematics Subject Classification: 14G40, 58J52, 20G05, 20G10, 14M17 


\section{Contents}

1 Introduction 2

2 Flag Varieties 4

3 Equivariant Ray-Singer torsion 10

\begin{tabular}{|lll}
4 A submersion formula for the analytic torsion & 11
\end{tabular}

5 The torsion of generalized flag manifolds 13

6 Description of the $L^{2}$-metric 21

$\begin{array}{lll}7 & \text { The main result } & 22\end{array}$

\begin{tabular}{|lll}
\hline & The height of generalized flag varieties & 27
\end{tabular} 


\section{Introduction}

One nice application of the classical Lefschetz fixed point formula is a geometric interpretation of the Weyl character formula for irreducible representations of a compact Lie group $G_{c}$, found by Atiyah and Bott [AB]. Namely, choose a maximal torus $T_{c}$ of $G_{c}$, fix a weight $\lambda$ and consider the line bundle associated to the $T_{c}$-representation of weight $\lambda$ over the flag variety $G_{c} / T_{c}$. The action of $G_{c}$ on $G_{c} / T_{c}$ induces an action on the cohomology vector spaces of this line bundle. The Lefschetz fixed point formula provides a formula for the character of this $G_{c}$-representation as a sum over the fixed point set. The fixed point set can be identified with the Weyl group of $G_{c}$, and the resulting formula is the classical Weyl character formula.

The purpose of this article is to investigate the analogous problem in the context of Arakelov geometry. Instead of compact Lie groups, we consider Chevalley schemes $G$ over Spec $\mathbf{Z}$. For a technical reason, we have to exclude factors of type $G_{2}, F_{4}$ and $E_{8}$. The generalized flag varieties associated to $G$ are acted upon by a maximal split torus of $G$. We apply to them the analogue of the fixed point formula in Arakelov geometry KR2 due to Roessler and one of the authors. If $V_{\mu}$ denotes the weight space to the weight $\mu$ in the cohomology representation, then the classical Lefschetz formula computes the character $\sum_{\mu \text { weight }} \mu \operatorname{dim} V_{\mu}$. In the Arakelov situation, the cohomology is a $\mathbf{Z}$-module whose tensor product with $\mathbf{C}$ carries an Hermitian metric, and the fixed point formula computes the arithmetic character

$$
\sum_{\mu \text { weight }} \mu \log \frac{\operatorname{covol} V_{\text {free }, \mu}}{\# V_{\text {tor }, \mu}} .
$$

with $V_{\text {free }}, V_{\text {tor }}$ indicate the free and torsion part, respectively. Having chosen a maximal split torus $T \subseteq G$ we have a canonical maximal compact subgroup $G_{c}$ in $G(\mathbf{C})$. Our metric on the cohomology is $G_{c}$-invariant and thus determined up to a factor. The arithmetic character of the alternating sum of the cohomology modules is then expressed in terms of roots and the Weyl group of $G_{c}$.

The resulting formula (up to the determination of the $L^{2}$-metric) turns out to be the Jantzen sum formula [122, p. 311] which has been proven originally using solely methods from representation theory and algebraic geometry. In contrast, in the proof presented here we mostly have to deal with the differential geometry of Laplace operators and we use only the representation theory of compact Lie groups. The arithmetic geometry is reduced to that of the fixed point scheme which consists of copies of Spec $\mathbf{Z}$.

We have to compute the equivariant holomorphic Ray-Singer torsion associated to vector bundles on complex homogeneous spaces. This is based on the formula for the Ray-Singer torsion for Hermitian symmetric spaces which has been determined by explicitly describing the zeta function defining the torsion by one of the authors [K2]. Using the arithmetic Lefschetz formula or a formula due to Ma Ma2, we can compare the analytic torsion of the base, of the total space and of the fiber of a fibration. Now we consider a tower of fibrations of complex 
homogeneous spaces whose fibers are Hermitian symmetric. The lowest base is a point and the total space is the full flag space. By an induction procedure, we get a formula for the torsion on any full flag space. Using again one fibration over an arbitrary complex homogeneous base and with total space and fiber being full flag spaces, we get an expression for the analytic torsion for that base.

In the last chapter, we use the Jantzen sum formula for the arithmetic character to derive formulae for the global height $h(X, \bar{L})$ of ample line bundles $\bar{L}$ over any (generalized) flag variety $X$ (now including the types $G_{2}, F_{4}, E_{8}$ ). The global height is defined using characteristic classes in Arakelov geometry.

The global height of generalized flag varieties has already been investigated in numerous cases using Arakelov intersection theory. There are explicit formulae for projective spaces due to Bost, Gillet and Soulé [BoGS] and for evendimensional quadrics by Cassaigne and Maillot [CM, Cor. 2.2.10]. Maillot [M] and Tamvakis [T1] found algorithms for the height of Grassmannians, leading to an explicit formula in the case $G(2, n)$ [T1]. Tamvakis also found combinatorial algorithms giving the height of generalized flag varieties of type $A_{n}$ [T2] and of Lagrangian Grassmannians [T3].

Let $\mathrm{Ht}$ denote the additive topological characteristic class associated to the power series

$$
\mathrm{Ht}(x):=\sum_{k=0}^{\infty} \frac{(-x)^{k}}{2(k+1)(k+1) !} .
$$

This is the Taylor expansion of the function $x \mapsto \frac{1}{2 x}\left(\log |x|-\Gamma^{\prime}(1)-\operatorname{Ei}(-x)\right)$ at $x=0$ with Ei being the exponential-integral function. Let $\overline{\mathcal{L}}$ be an equivariant line bundle on $G / P$, ample over $\mathbf{C}$. We construct in a simple way a virtual holomorphic vector bundle $y$ on $G_{\mathbf{C}} / P_{\mathbf{C}}$ such that the height with respect to $\overline{\mathcal{L}}$ is given by

$$
h(G / P, \overline{\mathcal{L}})=(n+1) ! \int_{G_{\mathbf{C}} / P_{\mathbf{C}}} \operatorname{Ht}(y) e^{c_{1}(L)}
$$

(Theorem 8.1). Also we give explicit formulae for the height in terms of roots and the Weyl group of $G_{c}$. From these formulae, we derive some simple properties of heights on generalized flag varieties.

Our method to compute the height is similar to the approach given in [KR3] by Roessler and one of the authors. Still, it is only an application of the classical Jantzen sum formula combined with a relation between the height and the asymptotic of the covolume of the cohomology of $\bar{L}^{m}$ for large $m \in \mathbf{N}$ which is described in SABK, VIII.2.3]. The proof does not make direct use of the Lefschetz fixed point formula in Arakelov geometry.

If one considers just the special case of Hermitian symmetric spaces, chapters 3-6 are not necessary for the proofs in chapters 7 and 8 . In particular the computation of the analytic torsion for generalized flag varieties in these chapters is already known in the case of Hermitian symmetric spaces ( $\mathrm{K} 2]$ ).

Furthermore, the full main result of [K2] is not needed in this article. In chapters 
3-6 we apply it only in the very special case of actions with isolated fixed points. In this case, the proof in $\mathrm{K} 2$ might be replaced by a considerably shorter one similar to the proof of [K3, Lemma 13].

This article is a part of the habilitation thesis of the second author.

Acknowledgements The authors wish to thank Gerd Faltings, Günter Harder, Jens-Carsten Jantzen, Damian Roessler and Matthias Weber for many valuable discussions and comments. We are also very grateful to the referees for their detailed comments.

\section{$2 \quad$ Flag Varieties}

In this chapter we recall the definition and some properties of the flag variety of a Chevalley group over $\mathbf{Z}$. Further on we describe the fixed point scheme under the action of some multiplicative subgroup scheme.

Notations: We denote the category of schemes resp. sets by Scheme resp. Set. For any scheme $S$ we denote by Scheme $_{S}$ the category of $S$-schemes. We identify a $S$-scheme by the functor from $\overline{\text { Scheme }_{S}}$ to Set which it represents. If $X$ and $S$ are schemes we set $X_{S}:=X \times S$ and consider it as an $S$-scheme. If $S=\operatorname{Spec} A$ is affine we also write $X_{A}$. For $A=\mathbf{C}$ we also denote by $X_{\mathbf{C}}$ the complex analytic variety $X(\mathbf{C})$. For a $\mathbf{Z}$-module $M$ and $\operatorname{ring} A$ we set $M_{A}:=M \otimes A$. Lie algebras associated to smooth group schemes shall be denoted by the corresponding german letter.

Let $G$ be a semisimple Chevalley group of rank $r$. This is a smooth affine group scheme over Spec $\mathbf{Z}$ with connected semisimple groups as geometric fibres. A closed subgroup scheme of $G_{S}$ is called a Borel subgroup, resp. a parabolic subgroup, if it is smooth and of finite presentation over $S$ and a Borel subgroup, resp. a parabolic subgroup, in every geometric fibre.

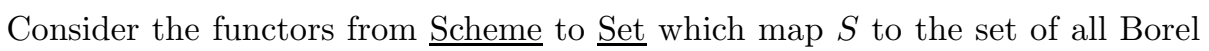
subgroups, resp. parabolic subgroups, of $G_{S}$. These functors are representable by smooth projective schemes $\operatorname{Bor}(G)$, resp. $\operatorname{Par}(G)$, over Spec Z SGA3, ch. XXVI, 3.3]. The group $G$ acts by conjugation on these schemes.

We fix now a maximal split torus $T \subseteq G$ with group of characters $X^{*}(T)$ and cocharacters $X_{*}(T)$, set of roots $\Sigma$ and Weyl group (of the root system) $W_{G}$. The adjoint action of $T$ on $\mathfrak{g}$ gives the weight decomposition in free $\mathbf{Z}$-modules:

$$
\mathfrak{g}=\mathfrak{t} \oplus \bigoplus_{\alpha \in \Sigma} \mathfrak{g}_{\alpha}
$$

Consider a parabolic subgroup $P$ containing $T$. Since the root spaces $\mathfrak{g}_{\alpha}$ are of rank one there is a subset $R \subseteq \Sigma$ with

$$
\mathfrak{p}=\mathfrak{t} \oplus \bigoplus_{\alpha \in R} \mathfrak{g}_{\alpha}
$$


The subset $R \subset \Sigma$ is closed (i.e. if $\alpha, \beta \in R$ and $\alpha+\beta \in \Sigma$ then $\alpha+\beta \in R$ ) and $R \cup-R=\Sigma$. We call such a subset of roots a parabolic subset.

Lemma 2.1 For each parabolic subset of roots $R \subset \Sigma$ there exists a unique parabolic subgroup $P(R)$ in $G$ containing $T$ such that $\mathfrak{p}(R)=\mathfrak{t} \oplus \bigoplus_{\alpha \in R} \mathfrak{g}_{\alpha}$.

Proof: The same proof as in [SGA3, XXII, 5.5.1]. Q.E.D.

For a $S$-group scheme $H$ acting on a $S$-scheme $Y$ we denote by $Y^{H}$ the functor of fixed points, i.e. for a $S$-scheme $S^{\prime}$ we have

$$
Y^{H}\left(S^{\prime}\right):=\left\{y \in Y\left(S^{\prime}\right) \mid \forall \phi: S^{\prime \prime} \rightarrow S^{\prime}, \forall h \in H\left(S^{\prime \prime}\right): h \phi^{*}(y)=\phi^{*}(y)\right\} .
$$

In the braces $\phi$ is an arbitrary $S$-morphism and $\phi^{*}$ the induced map on points.

Proposition 2.2 The functor morphism $\operatorname{Par}(G)^{T} \rightarrow \operatorname{Par}(G)$ is represented by the closed embedding of copies of Spec $\mathbf{Z}$ given by $P(R) \in \operatorname{Par}(G)(\mathbf{Z})$ :

$$
\bigsqcup_{R \subseteq \Sigma \text { parabolic }} \operatorname{Spec} \mathbf{Z} \rightarrow \operatorname{Par}(G) .
$$

Proof: Let $P$ be a parabolic subgroup over $S$. Then $P$ is its own normalizer ([SGA3, XXII, 5.8.5]), i.e. for any $S$-scheme $S^{\prime}$ and any $g \in G\left(S^{\prime}\right)$ with $\operatorname{int}(g)\left(P_{S^{\prime}}\right)=P_{S^{\prime}}$ we have $g \in P\left(S^{\prime}\right)$. Hence we have $P \in \operatorname{Par}(G)^{T}(S)$ iff $T_{S} \subset$ $P$. The parabolic subgroups containing $T_{S}$ are determined by their Lie algebra (SGA3, XXII, 5.3.5]). For $S$ connected we have $\mathfrak{p}=\mathfrak{t} \otimes \mathcal{O}_{S} \oplus \bigoplus_{\alpha \in R} \mathfrak{g}_{\alpha} \otimes \mathcal{O}_{S}$ for some parabolic subset of roots ( SGA3, XXVI, 1.4]), and hence $P=P(R)_{S}$. Since clearly $\operatorname{Par}(G)^{T}\left(S_{1} \sqcup S_{2}\right)=\operatorname{Par}(G)^{T}\left(S_{1}\right) \times \operatorname{Par}(G)^{T}\left(S_{2}\right)$ the claim follows. Q.E.D.

Let us fix an ordering $\Sigma=\Sigma^{+} \cup \Sigma^{-}$with base $\Pi$. A parabolic subset $R$ containing $\Sigma^{-}$, resp. its associated parabolic subgroup $P(R)$, is called "standard". For a standard parabolic subset $R$ we set type $(R)=\{\alpha \in \Pi \mid \alpha \in R \cap-R\}$. For $k$ a field every parabolic subgroup $P$ of $G_{k}$ is conjugate to an unique standard parabolic $P(R)$. We set type $(P):=\operatorname{type}(R)$. For general $S$ it is a locally constant function $s \rightarrow \operatorname{type}\left(P_{s}\right)$.

We have a type decomposition in open and closed subsets:

$$
\operatorname{Par}(G)=\bigsqcup_{\Theta \subseteq \Pi} \operatorname{Par}(G)_{\Theta}
$$

with $\operatorname{Par}(G)_{\Theta}$ classifying parabolic subgroups of type $\Theta$. Two parabolic groups have the same type if and only if they are locally conjugate w.r.t. the fpqc or étale topology ([SGA3, XXVI, 3.3]). Since parabolic subgroups are their own 
normalizer we see that $\operatorname{Par}(G)_{\Theta}$ represent also the fpqc- or étale-sheafification of the functor $S \rightarrow G(S) / P(S)$ for any $P \subseteq G$ of type $\Theta$. We write therefore $\operatorname{Par}(G)_{\Theta}=: G / P$. It is a connected scheme. We have $\operatorname{Par}(G)_{\emptyset}=\operatorname{Bor}(G)$ and $\operatorname{Par}(G)_{\Pi}=$ Spec $\mathbf{Z}$. Denoting the standard parabolic of type $\Theta$ by $P_{\Theta}$, Proposition 2.2 implies

Corollary 2.3 For $\Theta \subseteq \Pi$ we have

$$
\left(G / P_{\Theta}\right)^{T}=\bigsqcup_{w \in W_{G} / W_{\Theta}} \operatorname{Spec} \mathbf{Z}
$$

Here $W_{\Theta}$ is the subgroup generated by the reflections at roots in $\Theta$.

Remark : By [SGA3, XXVI, 3.16 (iii)] the fixed point scheme $(G / P)^{T}$ is finite étale over Spec $\mathbf{Z}$, from that Corollary 2.3 also follows.

We consider now a finite diagonalisable closed subgroup scheme $\mu \subseteq T$. This means we fix a finite abelian group $M$ and a surjective map $\psi: X^{*}(T) \rightarrow$ $M$. Then $\mu \simeq \operatorname{Spec} \mathbf{Z}[M]$ with comultiplication given by $\mathbf{Z}[M] \rightarrow \mathbf{Z}[M] \otimes$ $\mathbf{Z}[M], m \mapsto m \otimes m$. We assume now the following property for $\mu$ :

Definition 2.4 A finite diagonalisable closed subgroup scheme $\mu \subseteq T$ has the property (Reg) iff the restriction of $\psi$ to the set of roots $\Sigma$ is injective.

Proposition 2.5 If $\mu \subseteq T$ has property (Reg) then $\operatorname{Par}(G)^{\mu}=\operatorname{Par}(G)^{T}$.

Proof: Take $P \in \operatorname{Par}(G)(S)$. We have to show that $P$ is fixed by $\mu_{S}$ iff it is fixed by $T_{S}$. For this we can assume $G$ to be adjoint. From [SGA3, XXII, 5.3.3] it follows that $P$ is invariant under the action of $T_{S}$ resp. $\mu_{S}$ iff the same is true for $\mathfrak{p}$. It is a general property of a diagonalisable group scheme acting linearly on a module $M$ with weight decomposition $M=\bigoplus_{\alpha} M_{\alpha}$, that a submodule $N \subseteq M$ is invariant iff $N=\bigoplus_{\alpha}\left(N \cap M_{\alpha}\right)$. Since by property (Reg) the weight decompositions of $\mathfrak{g}$ with respect to $\mu$ and $T$ coincide the claim follows. Q.E.D.

Let $\langle\cdot, \cdot\rangle: X^{*}(T) \times X_{*}(T) \rightarrow \mathbf{Z}$ denote the natural pairing. For a coroot $\alpha^{\vee}$ we set $H_{\alpha}:=d \alpha^{\vee}(1) \in \mathfrak{t}$. For each $\alpha \in \Sigma$ there is a homomorphism $x_{\alpha}: \mathbf{G}_{a} \rightarrow G$ such that $d x_{\alpha}:$ Lie $\mathbf{G}_{a} \rightarrow \mathfrak{g}_{\alpha}$ induces an isomorphism. This map is unique up to a sign change. We can choose the maps $x_{\alpha}$ in such a way that with $X_{\alpha}:=d x_{\alpha}(1)$ we get a Chevalley base $\left\{X_{\alpha}, H_{\mu} \mid \alpha \in \Sigma, \mu \in \Pi\right\}$ of $\mathfrak{g} \otimes \mathbf{C}$. For $G$ simply connected it is actually a base of $\mathfrak{g}$. There is a unique involution $\tau$ acting on $G$ (J2, Corollary II.1.16 and proof]) with:

1) $\tau_{\mid T}: t \mapsto t^{-1} \quad$ 2) $\tau \circ x_{\alpha}(\cdot)=x_{-\alpha}(-\cdot)$.

It is independent from the consistent choice above. 
We can now define a maximal compact subgroup $G_{c}$ of $G_{\mathbf{C}}$ :

$$
G_{c}:=\left(G_{\mathbf{C}}\right)^{\tau \circ \iota} .
$$

Here $\iota$ denotes the complex conjugation.

Lemma 2.6 The group $N_{G}(T)(\mathbf{Z}) \cap G_{c}$ acts on $T$ by conjugation through the full Weyl group.

Proof: For each root $\alpha$ set $n_{\alpha}=x_{\alpha}(1) x_{-\alpha}(-1) x_{\alpha}(1)$. By [ ]2, p.176] we have $n_{\alpha} \in N_{G}(T)(\mathbf{Z})$ and it acts on $T$ by the reflection at $\alpha$. Since $\tau\left(x_{\alpha}(1) x_{-\alpha}(-1) x_{\alpha}(1)\right)=$ $x_{-\alpha}(-1) x_{\alpha}(1) x_{-\alpha}(-1)$ it is only an easy calculation in $S L_{2}$ to check $\tau\left(n_{\alpha}\right)=n_{\alpha}$ [J2, p.176]. Q.E.D.

We can use the maximal compact group $G_{c}$ to endow the complex flag variety $(G / P)_{\mathbf{C}}$ with a canonical hermitian metric which fits nicely with the integral structure. Fix a standard parabolic subgroup $P \subseteq G$. Setting $K:=P_{\mathbf{C}} \cap G_{c}$ the inclusion induces an isomorphism $(\mathfrak{g} / \mathfrak{p}) \otimes \mathbf{C} \simeq \mathfrak{g}_{c} / \mathfrak{k}$. For $\alpha$ a weight in $\mathfrak{g} / \mathfrak{p}$ it maps the integral generator of the $\alpha$-weight space $X_{\alpha}$ to $X_{\alpha}-X_{-\alpha}$. The negative of the Killing form $\kappa(\cdot, \cdot)$ induces a positive definite real bilinear form $(\cdot, \cdot)$ on $\mathfrak{m}:=\mathfrak{g}_{c} / \mathfrak{k}$. Denote the complex structure on $\mathfrak{m}$ by $J$. Set $\mathfrak{m}^{1,0}:=\{X \in$ $\mathfrak{m} \otimes \mathbf{C} \mid J X=i X\}$ and define $X^{1,0}:=(X-i J X) / 2$ for $X \in \mathfrak{m}$. The metric on $\mathfrak{m}$ induces a $G_{c}$-invariant Hermitian metric on $\mathfrak{m}^{1,0}$ and thus an Hermitian metric $(\cdot, \cdot)_{B}$ on $(G / P)_{\mathbf{C}}$. Using the Killing form we get a norm on $(\mathfrak{t} \otimes \mathbf{R})^{\vee} \cong$ $X^{*}(T) \otimes \mathbf{R}$. We get (using [Hu, p.37])

$$
\left(X_{\alpha}, X_{\alpha}\right)=-\kappa\left(X_{\alpha}-X_{-\alpha}, X_{\alpha}-X_{-\alpha}\right)=2 \kappa\left(X_{\alpha}, X_{-\alpha}\right)=\frac{4}{\|\alpha\|^{2}}
$$

and thus $\left\|X_{\alpha}^{1,0}\right\|_{B}^{2}=\frac{2}{\|\alpha\|^{2}}$. By corollary 2.3 the normal bundle of $(G / P)^{T}$ is canonically isomorphic to the restriction of the relative tangent bundle $T f$ to $(G / P)^{T}$, where $f: G / P \rightarrow$ Spec $\mathbf{Z}$ is the structure morphism. Using Lemma 2.6 and denoting by $\Psi \subseteq \Sigma^{+}$the set of weights in $\mathfrak{g} / \mathfrak{p}$, the above calculation gives

Lemma 2.7 For $\alpha \in \Psi$ the generators of the $\alpha$-weight spaces of the normal bundle of $(G / P)^{T}$ have squarelength $\frac{2}{\|\alpha\|^{2}}$ on every component.

Let $P$ be a standard parabolic subgroup. For $\lambda \in X^{*}(P)$ we define an invertible sheaf $\mathcal{L}_{\lambda}$ on $G / P$ by

$\mathcal{L}_{\lambda}(U)=\left\{f \in \mathcal{O}\left(\pi^{-1} U\right) \mid f(x p)=\lambda(p)^{-1} f(x) \quad \forall x \in\left(\pi^{-1} U\right)(S), p \in P(S)\right.$ and $\left.\forall S\right\}$.

Here $\pi: G \rightarrow G / P$ is the canonical map and $U \subseteq G / P$ an open subset. Considering $X^{*}(P)$ as a subgroup of $X^{*}(B)$ we have a canonical isomorphism $H^{*}\left(G / P, \mathcal{L}_{\lambda}\right) \simeq H^{*}\left(G / B, \mathcal{L}_{\lambda}\right)$. Hence in the following we consider only the 
"full flag variety". The groups $H^{*}(\lambda):=H^{*}\left(G / B, \mathcal{L}_{\lambda}\right)$ are finitely generated Z-modules with an algebraic action of $G$. We use the "dotted action" of the Weyl group: $w \cdot \mu=w(\mu+\rho)-\rho$. Here $\rho=\frac{1}{2} \sum_{\alpha \in \Sigma^{+}} \alpha$. Let $I_{+}$denote the set of dominant weights w.r.t. $\Sigma^{+}$. For the action of $G_{\mathbf{Q}}$ on $H^{*}(w \cdot \lambda)_{\mathbf{Q}}$ we have, denoting the irreducible representation of highest weight $\lambda$ by $V_{\rho+\lambda}$ and by $l(w)$ the length of a $w \in W_{G}$ :

Theorem 2.8 (Borel-Weil-Bott): a) If $\lambda+\rho \in I_{+}$but $\lambda \notin I_{+}$, then

$$
H^{*}(w \cdot \lambda)_{\mathbf{Q}}=0 \quad \text { for all } w \in W_{G} .
$$

b) If $\lambda \in I_{+}$then we have for all $w \in W_{G}$ and all $i \in \mathbf{N}_{0}$ :

$$
H^{i}(w \cdot \lambda)_{\mathbf{Q}} \simeq\left\{\begin{array}{cc}
V_{\rho+\lambda} & \text { if } i=l(w) \\
0 & \text { otherwise. }
\end{array}\right.
$$

For an abelian group $M$ we denote by $M_{\text {tor }}$ its torsion and set $M_{\text {free }}=M / M_{\text {tor }}$. Then $H^{i}(w \cdot \lambda)_{\text {free }}$ is a lattice in $H^{i}(w \cdot \lambda)_{\mathbf{Q}}$. The free part of the following Proposition is due to Andersen [A1, 2.10], the torsion part is a remark of Faltings. We set $X=G / B$ and $n+1=\operatorname{dim} X$.

Proposition 2.9 Let $\lambda$ be an arbitrary weight. For all $i \in \mathbf{Z}$ there are perfect pairings of $G$-modules

$$
\begin{gathered}
H^{i}(\lambda)_{\text {free }} \times H^{n-i}(-\lambda-2 \rho)_{\text {free }} \rightarrow \mathbf{Z} \\
H^{i}(\lambda)_{\text {tor }} \times H^{n+1-i}(-\lambda-2 \rho)_{\text {tor }} \rightarrow \mathbf{Q} / \mathbf{Z} .
\end{gathered}
$$

Proof: Applying Grothendieck duality Ha2 to the smooth and proper structure morphism $f: X \rightarrow$ Spec $\mathbf{Z}$ gives an isomorphism in the derived category of bounded complexes of abelian groups

$$
R \Gamma\left(\mathcal{L}_{-\lambda-2 \rho}\right)[n] \stackrel{\sim}{\rightarrow} R \operatorname{Hom}_{\mathbf{Z}}\left(R \Gamma\left(\mathcal{L}_{\lambda}\right), \mathbf{Z}\right) .
$$

The right hand side is a composition of derived functors and hence the limit of a (in this case: second quadrant) spectral sequence $\left(E_{r}^{i, j}\right)$, with $E_{2}^{i, j}=$ $\operatorname{Ext}_{\mathbf{Z}}^{j}\left(H^{-i}(\lambda), \mathbf{Z}\right)$. Since $\operatorname{Ext}_{\mathbf{Z}}^{j}(\cdot, \cdot)=0 \forall j \geq 2$ the spectral sequence degenerates at $E_{2}$, and we get exact sequences

$$
0 \rightarrow \operatorname{Ext}_{\mathbf{Z}}^{1}\left(H^{j}(\lambda), \mathbf{Z}\right) \rightarrow H^{n+1-j}(-\lambda-2 \rho) \rightarrow \operatorname{Hom}\left(H^{j-1}(\lambda), \mathbf{Z}\right) \rightarrow \mathbf{0} .
$$

The group $\operatorname{Ext}_{\mathbf{Z}}^{1}\left(H^{j}(\lambda), \mathbf{Z}\right)=\operatorname{Ext}_{\mathbf{Z}}^{1}\left(H^{j}(\lambda)_{\text {tor }}, \mathbf{Z}\right) \simeq \operatorname{Hom}\left(H^{j}(\lambda)_{\text {tor }}, \mathbf{Q} / \mathbf{Z}\right)$ is torsion and $\operatorname{Hom}\left(H^{j-1}(\lambda), \mathbf{Z}\right)$ is torsionfree. The claim follows. Q.E.D.

For a $T$-module $A$, which is a finite abelian group, we set char $A:=-\sum_{\mu} \mu \log \# A_{\mu}$. 
Corollary 2.10 char $H^{i}(\lambda)_{\text {tor }}=\operatorname{char} H^{n+1-i}\left(w_{0} \cdot \lambda\right)_{\text {tor }}$.

Proof: For $n_{0} \in N_{G}(T)(\mathbf{Z})$ acting on $T$ by $w_{0}$ we consider the automorphism $\widetilde{\tau}:=\operatorname{Int}\left(n_{0}\right) \circ \tau$. This automorphism respects $B$, acts therefore on $G / B$, and puts $\mathcal{L}_{\lambda}$ to $\mathcal{L}_{-w_{0} \lambda}$. Hence it induces an isomorphism $H^{i}(\lambda) \stackrel{\sim}{\rightarrow} H^{i}\left(-w_{0} \lambda\right)$, giving the equality char $\operatorname{Hom}\left(H^{i}(\lambda)_{\text {tor }}, \mathbf{Q} / \mathbf{Z}\right)=$ char $H^{i}\left(-w_{0} \lambda\right)_{\text {tor. }}$. The claim follows from the Proposition. Q.E.D.

We assume in the following $\lambda$ to be dominant. We choose a generator $v_{w . \lambda}$ of the $\lambda$-weight space of $H^{l(w)}(w \cdot \lambda)_{\text {free }}$. By irreducibility there is a unique $G_{c^{-}}$-invariant hermitian metric $(\cdot, \cdot)_{\tau}$ on $H^{l(w)}(w \cdot \lambda)_{\mathbf{C}}$ with $\left(v_{w . \lambda}, v_{w . \lambda}\right)_{\tau}=1$. Its restriction to $H^{l(w)}(w \cdot \lambda)_{\mathbf{R}}$ is a $\tau$-contravariant form ([01, p. 35]), i.e. $\left(g v, v^{\prime}\right)_{\tau}=$ $\left(v, \tau(g)^{-1} v^{\prime}\right)_{\tau}$, and was studied by Jantzen [J1 and Andersen [A1, p. 515]. By theorem 2.8 above we have for any $w \in W$ and for $w_{0} \in W$ the longest element an unique isomorphism:

$$
T_{w_{0}}: H^{l(w)}(w \cdot \lambda)_{\mathbf{Q}} \rightarrow H^{l\left(w_{0} w\right)}\left(w_{0} w \cdot \lambda\right)_{\mathbf{Q}}
$$

with $T_{w_{0}}\left(v_{w \cdot \lambda}\right)=v_{w_{0} w \cdot \lambda}$.

Proposition 2.11 The lattices $T_{w_{0}}\left(H^{l(w)}(w \cdot \lambda)_{\text {free }}\right)$ and $H^{l\left(w_{0} w\right)}\left(w_{0} w \cdot \lambda\right)_{\text {free }}$ are dual w.r.t. the pairing $(\cdot, \cdot)_{\tau}$.

Proof: This follows immediately from the explicit presentation of a contravariant form in [A1, p.515]. For convenience of the reader we recall it. For $n_{0} \in N_{G}(T)(\mathbf{Z})$ acting on $T$ by $w_{0}$ consider as above $\widetilde{\tau}:=\operatorname{Int}\left(n_{0}\right) \circ \tau$, inducing an isomorphism $\phi: H^{l(w)}(w \cdot \lambda) \stackrel{\sim}{\rightarrow} H^{l(w)}\left(-w_{0}(w \cdot \lambda)\right)$. We set $\psi:=n_{0}^{-1} \circ \phi$. It is independent of the choice of $n_{0}$ and $\psi(g v)=\tau(g) \psi(v)$. Using Serre duality pairing, for $v, v^{\prime} \in H^{l(w)}(w \cdot \lambda)_{\mathbf{Q}}$ we set :

$$
\beta\left(v, v^{\prime}\right)=\left(T_{w_{0}}(v), \psi\left(v^{\prime}\right)\right) .
$$

Note that $\beta\left(v_{w . \lambda}, v_{w . \lambda}\right)= \pm 1$. We have $\beta\left(g v, v^{\prime}\right)=\beta\left(v, \tau(g)^{-1} v^{\prime}\right)$. Hence it defines a non trivial $\tau$-contravariant form on $H^{l(w)}(w \cdot \lambda)_{\mathbf{Q}}$. We denote by $T_{w_{0} *}(\beta)$ the transported form on $H^{l\left(w_{0} w\right)}\left(w_{0} w \cdot \lambda\right)_{\mathbf{Q}}$. Then w.r.t. this form $H^{l\left(w_{0} w\right)}\left(w_{0} w \cdot \lambda\right)_{\text {free }}$ and $T_{w_{0}}\left(H^{l(w)}(w \cdot \lambda)_{\text {free }}\right)$ are dual lattices, as Serre duality induces a perfect pairing on $H_{\text {free }}^{\bullet}$ (Proposition 2.9). On the other hand by uniqueness of normalized $\tau$-contravariant forms we have $T_{w_{0} *}(\beta)= \pm(\cdot, \cdot)_{\tau}$. Q.E.D.

Take $w_{\lambda}$ in the $\lambda$-weight space of $V_{\rho+\lambda}$ and $w_{-\lambda}$ in the $-\lambda$-weight space of $V_{\rho+\lambda}^{\vee}$ with $\left\langle w_{\lambda}, w_{-\lambda}\right\rangle=1$, and consider the associated matrix coefficient $c_{w_{\lambda}, w_{-\lambda}}(g)=$ $\left\langle w_{\lambda}, g w_{-\lambda}\right\rangle$. The following result shall be used to refine our results in the case of positive line bundles:

Proposition 2.12 The function $c_{w_{\lambda}, w_{-\lambda}}$ is a generator of the $\lambda$-weight space of $H^{0}(\lambda)$. 
Proof: It is clear that $c_{w_{\lambda}, w_{-\lambda}}$ lies in the $\lambda$-weight space of $H^{0}(\lambda)_{\mathbf{Q}}$. Since $c_{w_{\lambda}, w_{-\lambda}}(1)=1$ it is enough to show that it defines a regular function on $G$. We can do this in two ways:

1) We can realize $V_{\rho+\lambda}, V_{\rho+\lambda}^{\vee}$ and the natural pairing between them by $H^{0}(\lambda)_{\mathbf{Q}}$, $H^{l\left(w_{0}\right)}(-\lambda-2 \rho)_{\mathbf{Q}}$ and the Serre duality pairing. By Proposition 2.9 this defines already a perfect pairing over $\mathbf{Z}$. Hence we can take appropriate elements in the integral structure for $w_{\lambda}$ and $w_{-\lambda}$. Therefore $c_{w_{\lambda}, w_{-\lambda}}$ is a regular function on $G$.

2) Using [J2, Proposition I.10.12] we have to show that $\mu\left(c_{w_{\lambda}, w_{-\lambda}}\right) \in \mathbf{Z}$ for all distributions $\mu \in \operatorname{Dist}(G)$. Almost by definition we have $\mu\left(c_{w_{\lambda}, w_{-\lambda}}\right)=$ $\left\langle\mu w_{\lambda}, w_{-\lambda}\right\rangle$. By [J2, p. 185] Dist $(G)$ is the subalgebra of $U(\mathfrak{g})$ generated by $X_{\alpha}^{n} / n$ ! with $\alpha \in \Sigma$ and $n \in \mathbf{N}$, and by all $\left(\begin{array}{c}H \\ m\end{array}\right)$ with $H \in \mathfrak{t}$ and $m \in \mathbf{N}$. There is an integral PBW-decomposition $U(\mathfrak{g})=U^{-} U^{0} U^{+}$with $U^{-}=\left\langle X_{\alpha}^{n} / n !\right| \alpha \in$ $\left.\Sigma^{-}, n \in \mathbf{N}\right\rangle, U^{+}=\left\langle X_{\alpha}^{n} / n ! \mid \alpha \in \Sigma^{+}, n \in \mathbf{N}\right\rangle$ and $U^{0}=\left\langle\left(\begin{array}{c}H \\ m\end{array}\right) \mid H \in \mathfrak{t}, m \in \mathbf{N}\right\rangle$. Since the vectors $w_{\lambda}$ and $w_{-\lambda}$ are highest weight vectors w.r.t. $B$ resp. the opposite Borel subgroup $B^{-}$one has only to consider $\mu \in U^{0}$. Considering $U^{0}$ as a polynomial algebra on $X^{*}(T)$ we get $\mu\left(c_{w_{\lambda}, w_{-\lambda}}\right)=\mu(\lambda)$. Since $\mathfrak{t}=$ $\left\{d \phi(1) \mid \phi \in X_{*}(T)\right\}$ we see that the polynomials $\left(\begin{array}{c}H \\ m\end{array}\right)$ take integral values on $X_{*}(T)$. The claim follows. Q.E.D.

\section{Equivariant Ray-Singer torsion}

In this section we repeat the definition of the equivariant Ray-Singer analytic torsion introduced in $[\mathrm{K} 1$. Let $M$ be a compact $n$-dimensional hermitian manifold with associated Kähler form $\omega$. Let $\bar{E}$ denote an hermitian holomorphic vector bundle on $M$ and let

$$
\bar{\partial}: \Gamma^{\infty}\left(\Lambda^{q} T^{* 0,1} M \otimes E\right) \rightarrow \Gamma^{\infty}\left(\Lambda^{q+1} T^{* 0,1} M \otimes E\right)
$$

be the Dolbeault operator. As in GS5], we equip $\mathfrak{A}^{0, q}(M, E):=\Gamma^{\infty}\left(\Lambda^{q} T^{* 0,1} M \otimes\right.$ $E)$ with the hermitian $L^{2}$-metric

$$
\left(\eta, \eta^{\prime}\right):=\int_{M}\left\langle\eta(x), \eta^{\prime}(x)\right\rangle \frac{\omega^{\wedge n}}{(2 \pi)^{n} n !} .
$$

Let $\bar{\partial}^{*}$ be the adjoint of $\bar{\partial}$ relative to this metric and let $\square_{q}:=\left(\bar{\partial}+\bar{\partial}^{*}\right)^{2}$ be the Kodaira-Laplacian acting on $\Gamma^{\infty}\left(\Lambda^{q} T^{* 0,1} M \otimes E\right)$ with spectrum $\sigma\left(\square_{q}\right)$. We denote by $\operatorname{Eig}_{\lambda}\left(\square_{q}\right)$ the eigenspace of $\square_{q}$ corresponding to an eigenvalue $\lambda$. Consider a holomorphic isometry $g$ of $M$ and assume given a holomorphic isometry $g^{E}: \bar{E} \rightarrow g^{*} \bar{E}$. The fixed point set of $g$ shall be denoted by $M^{g}$. This notation is chosen different from [KR2], KR3] to avoid other notational problems. The Dolbeault cohomology $H^{0, q}(M, E):=\operatorname{ker} \square_{q}$ shall be equipped with the restriction of the $L^{2}$-metric. The element $g$ induces an isometry $g^{*}$ of 
$H^{0, q}(M, E)$. Then the equivariant Quillen metric is defined via the zeta function

$$
Z_{g}(s):=\sum_{q>0}(-1)^{q+1} q \sum_{\substack{\lambda \in \sigma\left(\square_{q}\right) \\ \lambda \neq 0}} \lambda^{-s} \operatorname{Tr} g_{\mid \operatorname{Eig}_{\lambda}\left(\square_{q}\right)}^{*}
$$

for Re $s \gg 0$. Classically, this zeta function has a meromorphic continuation to the complex plane which is holomorphic at zero (Do ).

Definition 3.1 Set $\lambda_{g}(M, E):=\operatorname{det}_{g} H^{0, *}(M, E):=\bigotimes_{q}\left(\operatorname{det}_{g} H^{0,2 q}(M, E) \otimes\right.$ $\left.\operatorname{det}_{g} H^{0,2 q+1}(M, E)^{\vee}\right)$. The equivariant analytic torsion [K] is defined as

$$
T_{g}(M, \bar{E}):=Z_{g}^{\prime}(0) .
$$

For group actions with isolated fixed points on $M$, we consider the equivariant characteristic classes defined on $M^{g}$ which equal on every fixed point $p$

$$
\operatorname{Td}_{g}(E)_{\mid p}=\operatorname{det}\left(1-\left(g_{\mid p}^{E}\right)^{-1}\right)^{-1}
$$

and

$$
\operatorname{ch}_{g}(E)_{\mid p}=\operatorname{Tr} g_{\mid p}^{E} .
$$

These classes are defined in a more general context in [KR2, Th. 3.5].

\section{A submersion formula for the analytic torsion}

In this section we state a special case of a result due to Xiaonan Ma Ma2, namely for isolated fixed points. In this special case, it is also a direct consequence of the arithmetic Lefschetz formula in its formulation in [KR3, end of section 2, (b)]. We refer to [KR2, sections 3,4 and 6.2] for notation and definitions.

Consider a holomorphic Kähler fibration $f: M \rightarrow B$ of Kähler manifolds with fibre $Z$. Assume given an isometric automorphism $g$ of $f$ such that all fixed points are isolated. Take an equivariant holomorphic Hermitian vector bundle $\bar{E}$ on $M$ and assume that the dimension of the cohomology groups $H^{*}\left(Z, E_{\mid Z}\right)$ does not vary on $B$. For $\zeta \in S^{1}$ the short exact sequence

$$
0 \rightarrow T Z \rightarrow T M \rightarrow f^{*} T B \rightarrow 0
$$

decomposes over the fixed point set $M^{g}$ into $\zeta$-eigenspaces with respect to the action of $g$

$$
0 \rightarrow T Z_{\zeta} \rightarrow T M_{\zeta} \rightarrow f^{*} T B_{\zeta} \rightarrow 0 .
$$

Let $s_{\zeta}$ denote a non-zero element in $\operatorname{det} T M_{\zeta}$ and let $s_{\zeta}^{\prime} \in \operatorname{det} T Z_{\zeta} \otimes \operatorname{det} f^{*} T B_{\zeta}$ be induced from $s_{\zeta}$ by the sequence above. Consider the characteristic class $\operatorname{Td}_{g}$ 
as defined in [KR2, section 3.3]. We define the map $\widetilde{\mathrm{Td}}_{g}(\overline{T M}, \overline{T B}): M_{g} \rightarrow \mathbf{C}$ as

$$
\widetilde{\operatorname{Td}}_{g}(\overline{T M}, \overline{T B}):=\operatorname{Td}_{g}(T M) \sum_{\zeta \neq 1} \frac{1}{\zeta-1} \log \frac{\left\|s_{\zeta}\right\|^{2}}{\left\|s_{\zeta}^{\prime}\right\|^{2}}
$$

(see Ma2 for the general definition which corresponds to the definition in KR2, Th. 3.5] up to a sign). For our proof, we need to assume that $f$ extends over Spec $\mathbf{Z}$ to a flat projective map of arithmetic varieties $f: X \rightarrow Y$. We assume furthermore that $g$ corresponds to the action of a diagonalisable group scheme $\mu_{N}$ as in [KR2, section 4] and that $E$ extends to an equivariant locally free sheaf over $X$.

Consider the structure morphisms $f_{X}: X \rightarrow \operatorname{Spec} \mathbf{Z}, f_{Y}: Y \rightarrow \operatorname{Spec} \mathbf{Z}$ and endow the direct image sheafs $R f_{X *} E, R f_{*} E$ and $R f_{Y *}\left(R f_{*} E\right)$ over $\mathbf{C}$ with the $L^{2}$-metrics induced by Hodge theory. The direct image sheaf equipped with these metrics shall be denoted by $R f_{X *} \bar{E}$ etc. The Leray spectral sequence provides an isomorphism $R f_{X *} E \rightarrow R f_{Y *}\left(R f_{*} E\right)$. Consider over $\mathbf{C}$ the equivariant Knudsen-Mumford determinant lines $\lambda_{M, g}:=\operatorname{det}_{g}\left(H^{\cdot}(M, \bar{E})\right)^{-1}$ and $\lambda_{B, g}:=\operatorname{det}_{g}\left(\sum_{j, k}(-1)^{j+k} H^{j}\left(B, H^{k}\left(Z, \bar{E}_{\mid Z}\right)\right)\right)^{-1}$ and let $\sigma: \lambda_{B, g} \rightarrow \lambda_{M, g}$ denote the isomorphism between them induced by the Leray spectral sequence. Thus, $\log \|\sigma\|_{L^{2}, g}^{2}=\log \frac{\|s\|_{\lambda_{M}, g}^{2}}{\|\sigma s\|_{\lambda_{B}, g}^{2}}$ for non-vanishing $s \in \lambda_{M, g}$.

Theorem 4.1 The equivariant analytic torsions of $M, B$ and $Z$ are related by the equation

$$
\begin{aligned}
& \log \|\sigma\|_{L^{2}, g}^{2}-T_{g}(M, \bar{E}) \\
& \quad=-T_{g}\left(B, R f_{*} \bar{E}\right)-\int_{B^{g}} \operatorname{Td}_{g}(T B) T_{g}(Z, \bar{E})+\int_{M^{g}} \widetilde{\operatorname{Td}_{g}}(\overline{T M}, \overline{T B}) \operatorname{ch}_{g}(E) .
\end{aligned}
$$

As all fixed points are isolated in our case, it does not make any difference in this formula whether one considers classes $\operatorname{Td}_{g}(T B)$ or characteristic differential forms like $\operatorname{Td}_{g}(\overline{T B})$ as in [Ma2]. In [Ma2], Ma does not need the existence of models over Spec $\mathbf{Z}$.

Proof: We use the notations of [KR2, Th. 6.14]. Then the arithmetic Lefschetz formula applied subsequently to $f_{X}, f$ (in its generalization in KR3, end of section 2, (b)]) and to $f_{Y}$ yields

$$
\begin{aligned}
2 \widehat{\operatorname{deg}} \widehat{c h}_{\mu_{N}}\left(R f_{X *} \bar{E}\right)-T_{g}(M, \bar{E})=2 \widehat{\operatorname{deg}} \widehat{c h}_{\mu_{N}}\left(f_{X !} \bar{E}\right) \\
=2 \widehat{\operatorname{deg}} f_{X *}^{\mu_{N}}\left(\widehat{\operatorname{Td}}_{\mu_{N}}\left(\overline{T f_{X}}\right) \widehat{\operatorname{ch}}_{\mu_{N}}(\bar{E})\right)-\int_{M^{g}} \operatorname{Td}_{g}(T M) R_{g}(T M) \operatorname{ch}_{g}(E) \\
=2 \widehat{\operatorname{deg}} f_{Y *}^{\mu_{N}}\left[\widehat{\operatorname{Td}}_{\mu_{N}}\left(\overline{T f_{Y}}\right) f_{*}^{\mu_{N}}\left(\widehat{\operatorname{Td}}_{\mu_{N}}(\overline{T f}) \widehat{\operatorname{ch}}_{\mu_{N}}(\bar{E})\right)\right] \\
\quad+\int_{M^{g}} \widetilde{\operatorname{Td}}_{g}(\overline{T M}, \overline{T B}) \operatorname{ch}_{g}(E)-\int_{M^{g}} \operatorname{Td}_{g}(T M) R_{g}(T M) \operatorname{ch}_{g}(E)
\end{aligned}
$$




$$
\begin{aligned}
& =2 \widehat{\operatorname{deg}} f_{Y *}^{\mu_{N}}\left[\widehat { \operatorname { T d } } _ { \mu _ { N } } ( \overline { T f _ { Y } } ) \left(\widehat{\operatorname{ch}}_{\mu_{N}}\left(R f_{*} \bar{E}\right)-T_{g}(Z, \bar{E})\right.\right. \\
& \left.\left.+\int_{Z^{g}} \operatorname{Td}_{g}(T Z) R_{g}(T Z) \operatorname{ch}_{g}(E)\right)\right] \\
& +\int_{M^{g}} \widetilde{\mathrm{Td}_{g}}(\overline{T M}, \overline{T B}) \operatorname{ch}_{g}(E)-\int_{M^{g}} \operatorname{Td}_{g}(T M) R_{g}(T M) \operatorname{ch}_{g}(E) \\
& =2 \widehat{\operatorname{deg}} \widehat{\operatorname{ch}}_{\mu_{N}}\left(R f_{Y *}\left(R f_{*} \bar{E}\right)\right)-T_{g}\left(B, R f_{*} \bar{E}\right) \\
& +\int_{B^{g}} \operatorname{Td}_{g}(T B) R_{g}(T B) \operatorname{ch}_{g}\left(R f_{*} E\right) \\
& -\int_{B^{g}} \operatorname{Td}_{g}(\overline{T B}) T_{g}(Z, \bar{E})+\int_{B^{g}} \operatorname{Td}_{g}(T B) \int_{Z^{g}} \operatorname{Td}_{g}(T Z) R_{g}(T Z) \operatorname{ch}_{g}(E) \\
& +\int_{M^{g}} \widetilde{\mathrm{Td}}_{g}(\overline{T M}, \overline{T B}) \operatorname{ch}_{g}(\bar{E})-\int_{M^{g}} \operatorname{Td}_{g}(T M) R_{g}(T M) \operatorname{ch}_{g}(E) \\
& =2 \widehat{\operatorname{deg}} \widehat{\operatorname{ch}}_{\mu_{N}}\left(R f_{Y *}\left(R f_{*} \bar{E}\right)\right)-T_{g}\left(B, R f_{*} \bar{E}\right) \\
& -\int_{B^{g}} \operatorname{Td}_{g}(\overline{T B}) T_{g}(Z, \bar{E})+\int_{M^{g}} \widetilde{\mathrm{Td}}_{g}(\overline{T M}, \overline{T B}) \operatorname{ch}_{g}(\bar{E}) .
\end{aligned}
$$

Furthermore

$$
2 \widehat{\operatorname{deg}} \widehat{c h}_{\mu_{N}}\left(R f_{X *} \bar{E}\right)-2 \widehat{\operatorname{deg}} \widehat{c h}_{\mu_{N}}\left(R f_{Y *}\left(R \cdot f_{*} \bar{E}\right)\right)=\log \|\sigma\|_{L^{2}, g}^{2}
$$

by the Leray spectral sequence, which in particular identifies the torsion subgroups of the direct images. Thus we get the statement of the theorem. Q.E.D.

Remark. By $K$-theory, one could in fact reduce oneself to the case where the direct images are locally free and non-zero only in degree 0. Then one can consider the equivariant determinant lines over $\operatorname{Spec} \mathbf{Z}$ and identify them directly as graded direct sums of $\mathbf{Z}$-modules of rank one (up to a sign). Thus one could avoid the use of the spectral sequence entirely.

An arithmetic Lefschetz formula for more general fibrations (combined with a general arithmetic Grothendieck-Riemann-Roch theorem) would provide this formula not only for non-isolated fixed points, but for the torsion forms associated to a double fibration $M \rightarrow B \rightarrow S$ with a Kähler manifold $S$ as treated in [Ma1 in the non-equivariant case.

\section{The torsion of generalized flag manifolds}

Set $T_{c}:=G_{c} \cap T_{\mathbf{C}}$. We denote the roots of $K$ by $\Sigma_{K}$; in general, we shall denote the objects corresponding to subgroups of $G$ by writing this subgroup as an index. Then $M:=G_{\mathbf{C}} / P_{\mathbf{C}}$ is canonically isomorphic to $G_{c} / K$ and $\Sigma^{+}=\Psi \cup \Sigma_{K}^{+}$. Recall that we identify $\mathfrak{m}^{1,0}$ with the tangent space $T_{e} M^{1,0}$ and that the negative of the Killing form induces an Hermitian metric on $T M$. Fix $X_{0} \in \mathfrak{t}_{c}$ in the 
closure of the positive Weyl chamber such that its stabilizer with respect to the adjoint action of $G_{c}$ equals $K$. Then the metric $(\cdot, \cdot)_{X_{0}}$ on $\mathfrak{m}$ which equals for $X \in \mathfrak{m}_{\alpha}, Y \in \mathfrak{m}_{\beta}$

$$
(X, Y)_{X_{0}}=\left\{\begin{array}{cc}
0 & \alpha \neq \beta \\
(X, Y)_{B} \alpha\left(X_{0}\right) & \alpha=\beta
\end{array}\right.
$$

induces a Kähler metric on $M[\mathrm{~B}$, Ch. 8.D].

Set $\mathfrak{t}_{\text {reg }}:=\left\{X \in \mathfrak{t}_{c} \mid \alpha(X) \notin \mathbf{Z} \forall \alpha \in \Sigma\right\}$. For $X \in \mathfrak{t}_{c}$ let $e^{X} \in T_{c}$ denote the associated group element. We denote the $G_{c^{-}}$-representation with highest weight $\lambda$ by $V_{\rho+\lambda}^{G}$ and its character is denoted by $\chi_{\rho+\lambda}$. In general, for a weight $\lambda$ and $X \in \mathfrak{t}_{\text {reg }}$ we define $\chi_{\rho+\lambda}$ by the Weyl character formula

$$
\chi_{\rho+\lambda}\left(e^{X}\right):=\frac{\sum_{w \in W_{G}}(-1)^{l(w)} e^{2 \pi i(\rho+\lambda)(w X)}}{\prod_{\alpha \in \Sigma^{+}} 2 i \sin \pi \alpha(X)} .
$$

An irreducible $K$-representation $V_{\rho_{K}+\lambda}^{K}$ induces a $G_{c}$-invariant holomorphic vector bundle $E_{\rho_{K}+\lambda}^{K}$ on $M$. As $V_{\rho_{K}+\lambda}^{K}$ carries a $K$-invariant Hermitian metric which is unique up to a factor, we get corresponding $G_{c}$-invariant metrics on $E_{\rho_{K}+\lambda}^{K}$. Set $\Psi^{+}:=\left\{\alpha \in \Psi \mid\left\langle\alpha^{\vee}, \rho+\lambda\right\rangle \geq 0\right\}$ and $\Psi^{-}:=\left\{\alpha \in \Psi \mid\left\langle\alpha^{\vee}, \rho+\lambda\right\rangle<0\right\}$ with $\alpha^{\vee}=2 \alpha /\|\alpha\|^{2}$.

Define for $\phi \in \mathbf{R}$ and $\operatorname{Re} s>1$

$$
\zeta_{L}(s, \phi)=\sum_{k=1}^{\infty} \frac{e^{i k \phi}}{k^{s}} .
$$

The function $\zeta_{L}$ has a meromorphic continuation to the complex plane in $s$ which is holomorphic for $s \neq 1$. Set $\zeta_{L}^{\prime}(s, \phi):=\partial / \partial s\left(\zeta_{L}(s, \phi)\right)$. Let $P: \mathbf{Z} \rightarrow \mathbf{C}$ be a function of the form

$$
P(k)=\sum_{j=0}^{m} c_{j} k^{n_{j}} e^{i k \phi_{j}}
$$

with $m \in \mathbf{N}_{0}, n_{j} \in \mathbf{N}_{0}, c_{j} \in \mathbf{C}, \phi_{j} \in \mathbf{R}$ for all $j$. We define $P^{\text {odd }}(k):=$ $(P(k)-P(-k)) / 2$. Also we define as in $\mathrm{K2}$

$$
\begin{aligned}
\boldsymbol{\zeta} P & :=\sum_{j=0}^{m} c_{j} \zeta_{L}\left(-n_{j}, \phi_{j}\right), \\
\boldsymbol{\zeta}^{\prime} P & :=\sum_{j=0}^{m} c_{j} \zeta_{L}^{\prime}\left(-n_{j}, \phi_{j}\right), \\
\overline{\boldsymbol{\zeta}} P & :=\sum_{j=0}^{m} c_{j} \zeta_{L}\left(-n_{j}, \phi_{j}\right) \sum_{\ell=1}^{n_{j}} \frac{1}{\ell} .
\end{aligned}
$$




$$
\begin{aligned}
\operatorname{Res} P(p) & :=\sum_{\substack{j=0 \\
\phi_{j}=0 \bmod 2 \pi}}^{m} c_{j} \frac{p^{n_{j}+1}}{2\left(n_{j}+1\right)} \\
\text { and } \quad P^{*}(p) & :=-\sum_{\substack{j=0 \\
\phi_{j}=0 \bmod 2 \pi}}^{m} c_{j} \frac{p^{n_{j}+1}}{4\left(n_{j}+1\right)} \sum_{\ell=1}^{n_{j}} \frac{1}{\ell}
\end{aligned}
$$

for $p \in \mathbf{R}$. In particular, for $X \in \mathfrak{t}_{\text {reg }}$ we get (compare $[\mathrm{K} 2$, Th. 10, top of p. 108])

$$
\zeta \chi_{\rho+\lambda-k \alpha}\left(e^{X}\right)=\frac{\sum_{w \in W_{G}}(-1)^{l(w)} e^{2 \pi i(\rho+\lambda)(w X)}\left(e^{2 \pi i \alpha(w X)}-1\right)^{-1}}{\prod_{\beta \in \Sigma^{+}} 2 i \sin \pi \beta(X)} .
$$

Assume now that $M=G_{c} / K$ is an Hermitian symmetric space. Then the isotropy representation is irreducible, hence all $G_{c}$-invariant metrics coincide with $(\cdot, \cdot)_{B}$ up to a factor. Fix one metric associated to $X_{0}$ with stabilizer $K$. In [K2, section 11], the analytic torsion on $G_{c} / K$ has been calculated for vector bundles $\bar{E}_{\rho_{K}+\lambda}^{K}$ with $\lambda \in I_{+}$. We shall now extend this result to arbitrary $\lambda$.

Proposition 5.1 Assume that $G_{c} / K$ is Hermitian symmetric. Then the zeta function defining the analytic torsion of $\bar{E}_{\rho_{K}+\lambda}^{K}$ is given by

$$
\begin{aligned}
Z_{t}(s)= & -\sum_{\alpha \in \Psi^{+}} \alpha^{\vee}\left(X_{0}\right)^{s} \sum_{k>\left\langle\alpha^{\vee}, \rho+\lambda\right\rangle} \frac{\chi_{\rho+\lambda-k \alpha}(t)}{k^{s}\left(k-\left\langle\alpha^{\vee}, \rho+\lambda\right\rangle\right)^{s}} \\
& +\sum_{\alpha \in \Psi^{-}} \alpha^{\vee}\left(X_{0}\right)^{s} \sum_{k>-\left\langle\alpha^{\vee}, \rho+\lambda\right\rangle} \frac{\chi_{\rho+\lambda+k \alpha}(t)}{k^{s}\left(k+\left\langle\alpha^{\vee}, \rho+\lambda\right\rangle\right)^{s}} .
\end{aligned}
$$

Proof: According to [K2, (114)], the irreducible representations occurring as eigenspaces of the Laplacian acting on $\mathfrak{A}^{0, q}(M, E)$ are given by the infinitesimal characters $\rho+\lambda+k \alpha$ with $k>0$. Consider now the case $\left\langle\alpha^{\vee}, \rho+\lambda\right\rangle<0$ and assume $0<k<\left\langle-\alpha^{\vee}, \rho+\lambda\right\rangle / 2$. Let $S_{\alpha}$ denote the reflection of the weights by the hyperplane orthogonal to $\alpha$. Then the multiplicity of $V_{\rho+\lambda+k \alpha}$ cancels with that of $V_{S_{\alpha}(\rho+\lambda+k \alpha)}=V_{\rho+\lambda+\left(-k-\left\langle\alpha^{\vee}, \rho+\lambda\right\rangle\right) \alpha}$. In case $\left\langle\alpha^{\vee}, \rho+\lambda\right\rangle$ is even, the representation $V_{\rho+\lambda-\left\langle\alpha^{\vee}, \rho+\lambda\right\rangle \alpha / 2}$ vanishes. Also, the representation $V_{\rho+\lambda-\left\langle\alpha^{\vee}, \rho+\lambda\right\rangle \alpha}$ corresponds to the eigenvalue 0 , thus it does not contribute to the zeta function. Thus, $Z_{t}(s)$ is given by

$$
Z_{t}(s)=\sum_{\alpha \in \Psi} \alpha^{\vee}\left(X_{0}\right)^{s} \sum_{k>\max \left\{0,\left\langle-\alpha^{\vee}, \rho+\lambda\right\rangle\right\}} \frac{\chi_{\rho+\lambda+k \alpha}}{k^{s}\left(k+\left\langle\alpha^{\vee}, \rho+\lambda\right\rangle\right)^{s}}
$$

which equals the above formula after another application of $S_{\alpha}$ for $\alpha \in \Psi^{+}$. Q.E.D. 
Theorem 5.2 Assume that $G_{c} / K$ is Hermitian symmetric. Then the equivariant analytic torsion of $\bar{E}_{\rho_{K}+\lambda}^{K}$ is given by

$$
\begin{aligned}
& T_{t}\left(G_{c} / K, \bar{E}_{\rho_{K}+\lambda}^{K}\right)=-2 \sum_{\alpha \in \Psi} \zeta^{\prime} \chi_{\rho+\lambda-k \alpha}^{\text {odd }}-2 \sum_{\alpha \in \Psi} \chi_{\rho+\lambda-k \alpha}^{*}\left(\left\langle\alpha^{\vee}, \rho+\lambda\right\rangle\right) \\
& -\sum_{\alpha \in \Psi} \zeta \chi_{\rho+\lambda-k \alpha} \cdot \log \alpha^{\vee}\left(X_{0}\right)-\chi_{\rho+\lambda} \sum_{\alpha \in \Psi^{+}} \log \alpha^{\vee}\left(X_{0}\right) \\
& \quad-\sum_{\alpha \in \Psi^{+}} \sum_{k=1}^{\left\langle\alpha^{\vee}, \rho+\lambda\right\rangle} \chi_{\rho+\lambda-k \alpha} \cdot \log k+\sum_{\alpha \in \Psi^{-}} \sum_{k=1}^{\left\langle-\alpha^{\vee}, \rho+\lambda\right\rangle} \chi_{\rho+\lambda+k \alpha} \cdot \log k .
\end{aligned}
$$

Proof: According to [K2, Lemma 8] and [K2, (61),(63)], we find

$$
\begin{aligned}
Z_{t}^{\prime}(0)= & \sum_{\alpha \in \Psi^{+}}\left[-2 \boldsymbol{\zeta}^{\prime} \chi_{\rho+\lambda-k \alpha}^{\text {odd }}-2 \chi_{\rho+\lambda-k \alpha}^{*}\left(\left\langle\alpha^{\vee}, \rho+\lambda\right\rangle\right)\right. \\
& \left.-\sum_{k=1}^{\left\langle\alpha^{\vee}, \rho+\lambda\right\rangle} \chi_{\rho+\lambda-k \alpha} \cdot \log k\right] \\
& -\sum_{\alpha \in \Psi^{+}}\left[\boldsymbol{\zeta} \chi_{\rho+\lambda-k \alpha}-\sum_{k=1}^{\left\langle\alpha^{\vee}, \rho+\lambda\right\rangle} \chi_{\rho+\lambda-k \alpha}\right] \log \alpha^{\vee}\left(X_{0}\right) \\
& +\sum_{\alpha \in \Psi^{-}}\left[2 \boldsymbol{\zeta}^{\prime} \chi_{\rho+\lambda+k \alpha}^{\text {odd }}+2 \chi_{\rho+\lambda+k \alpha}^{*}\left(\left\langle-\alpha^{\vee}, \rho+\lambda\right\rangle\right)\right. \\
& \left.+\sum_{k=1}^{\left\langle-\alpha^{\vee}, \rho+\lambda\right\rangle} \chi_{\rho+\lambda+k \alpha} \cdot \log k\right]-\sum_{\alpha \in \Psi^{-}} \boldsymbol{\zeta} \chi_{\rho+\lambda-k \alpha} \log \alpha^{\vee}\left(X_{0}\right)
\end{aligned}
$$

As

$$
-\sum_{k=1}^{\left\langle\alpha^{\vee}, \rho+\lambda\right\rangle} \chi_{\rho+\lambda-k \alpha}=-\chi_{\rho+\lambda-\left\langle\alpha^{\vee}, \rho+\lambda\right\rangle \alpha}=\chi_{\rho+\lambda}
$$

for $\alpha \in \Psi^{+}$and

$$
\begin{gathered}
\boldsymbol{\zeta}^{\prime} \chi_{\rho+\lambda+k \alpha}^{\text {odd }}=-\boldsymbol{\zeta}^{\prime} \chi_{\rho+\lambda-k \alpha}^{\text {odd }}, \\
\chi_{\rho+\lambda+k \alpha}^{*}\left(\left\langle-\alpha^{\vee}, \rho+\lambda\right\rangle\right)=-\chi_{\rho+\lambda-k \alpha}^{*}\left(\left\langle\alpha^{\vee}, \rho+\lambda\right\rangle\right)
\end{gathered}
$$

we get the statement of the theorem. Q.E.D.

According to [K2, (103),(116)], for any generalized flag manifold the equation

$$
\int_{M^{t}} \operatorname{Td}_{t}(T M) R_{t}(T M) \operatorname{ch}_{t}\left(E_{\rho_{K}+\lambda}^{K}\right)=-\sum_{\alpha \in \Psi}\left[2 \zeta^{\prime} \chi_{\rho+\lambda-k \alpha}^{\text {odd }}+\bar{\zeta} \chi_{\rho+\lambda-k \alpha}^{\text {odd }}\right]
$$

holds. For fixed $X \in \mathfrak{t}_{\text {reg }}$, according to $\left[\mathbf{K} 2\right.$, eq. (66)] the characters $\chi_{\rho+\lambda-k \alpha}\left(e^{i X}\right)$ can be written as a linear combination of exponential functions in $k$. Polynomial functions in $k$ do not occur in this case. Thus, by definition $\chi_{\rho+\lambda-k \alpha}^{*}$ and $\overline{\boldsymbol{\zeta}} \chi_{\rho+\lambda-k \alpha}$ vanish. Hence, we get the following simpler formula for generic $X$ : 
Corollary 5.3 Assume that $M=G_{c} / K$ is Hermitian symmetric and choose $X \in \mathfrak{t}_{\text {reg. }}$. Then

$$
\begin{aligned}
& T_{t}\left(\left(M, g_{X_{0}}\right), \bar{E}_{\rho_{K}+\lambda}^{K}\right)-\int_{M^{t}} \operatorname{Td}_{t}(T M) R_{t}(T M) \operatorname{ch}_{t}\left(E_{\rho_{K}+\lambda}^{K}\right)= \\
& \quad-\sum_{\alpha \in \Psi} \zeta \chi_{\rho+\lambda-k \alpha} \cdot \log \alpha^{\vee}\left(X_{0}\right)-\chi_{\rho+\lambda} \sum_{\alpha \in \Psi^{+}} \log \alpha^{\vee}\left(X_{0}\right) \\
& \quad-\sum_{\alpha \in \Psi^{+}} \sum_{k=1}^{\left\langle\alpha^{\vee}, \rho+\lambda\right\rangle} \chi_{\rho+\lambda-k \alpha} \cdot \log k+\sum_{\alpha \in \Psi^{-}} \sum_{k=1}^{\left\langle-\alpha^{\vee}, \rho+\lambda\right\rangle} \chi_{\rho+\lambda+k \alpha} \cdot \log k .
\end{aligned}
$$

We say that a semisimple Lie group has tiny weights if none of its simple components is of type $G_{2}, F_{4}$ or $E_{8}$.

Theorem 5.4 Let $G_{\mathbf{C}}$ be a semisimple Lie group having tiny weights and let $M=G_{c} / K$ be an associated (generalized) flag manifold. Let $\bar{E}_{\rho_{K}+\lambda}^{K}$ be a $G_{c^{-}}$ invariant holomorphic Hermitian vector bundle on $M$ and fix a Kähler metric $g_{X_{0}}$ associated to $X_{0} \in \mathfrak{t}_{c}$ on $M$. Choose $X \in \mathfrak{t}_{\text {reg }}$ and set $t:=e^{X}$. Then the equivariant analytic torsion associated to the action of $t$ is given by

$$
\begin{aligned}
& T_{t}\left(\left(M, g_{X_{0}}\right), \bar{E}_{\rho_{K}+\lambda}^{K}\right)-\int_{M^{t}} \operatorname{Td}_{t}(T M) R_{t}(T M) \operatorname{ch}_{t}\left(E_{\rho_{K}+\lambda}^{K}\right)= \\
& \quad-\sum_{\alpha \in \Psi} \zeta \chi_{\rho+\lambda-k \alpha} \cdot \log \alpha^{\vee}\left(X_{0}\right)+C \chi_{\rho+\lambda} \\
& \quad-\sum_{\alpha \in \Psi^{+}} \sum_{k=1}^{\left\langle\alpha^{\vee}, \rho+\lambda\right\rangle} \chi_{\rho+\lambda-k \alpha} \cdot \log k+\sum_{\alpha \in \Psi^{-}} \sum_{k=1}^{\left\langle-\alpha^{\vee}, \rho+\lambda\right\rangle} \chi_{\rho+\lambda+k \alpha} \cdot \log k
\end{aligned}
$$

where the constant $C \in \mathbf{R}$ does not depend on $t$.

Our proof proceeds by constructing first a tower of fibrations of flag manifolds with total space a full flag manifold $G_{\mathbf{C}} / B_{\mathbf{C}}$, such that all fibers are Hermitian symmetric spaces. As the torsion is known for the fibers, we can deduce the value of the torsion for the total space of every fibration from the value on the base. Thus we finally get the value for the full flag manifold. Then we use the fibration $G_{\mathbf{C}} / B_{\mathbf{C}} \rightarrow G_{\mathbf{C}} / P_{\mathbf{C}}$ with both total space and fiber being full flag manifolds to compute the torsion for any flag manifold. We need first a Lemma and a Proposition:

Lemma 5.5 Let $G_{\mathbf{C}}$ be a reductive group having tiny weights. Then there is an ordering $\left(\alpha_{1}, \ldots, \alpha_{m}\right)$ of the base $\Pi$ such that with $\Theta_{j}:=\left\{\alpha_{1}, \ldots, \alpha_{j}\right\}, 0 \leq$ $j \leq m$, all quotients of subsequent parabolic subgroups $P_{\Theta_{j+1}} / P_{\Theta_{j}}, 0 \leq j<m$, are Hermitian symmetric spaces.

Proof: The only simple groups whose quotients by parabolic subgroups are never Hermitian symmetric spaces are the groups $G_{2}, F_{4}$ and $E_{8} \mathrm{He}$, Ch. X 
$\S 6.3]$. If $G_{\mathbf{C}}$ has tiny weights, then the Dynkin diagram of $\mathfrak{g}_{2}, \mathfrak{f}_{4}$ or $\mathfrak{e}_{8}$ can never occur as a subdiagram of the Dynkin diagram of $\mathfrak{g}_{\mathbf{C}}$. Thus, there is always a simple root $\alpha$ such that the quotient $G_{\mathbf{C}} / P_{\Pi \backslash\{\alpha\}}$ is Hermitian symmetric (namely, those $\alpha$ such that their coefficient in the highest root is 1, compare [ $\mathrm{HA}$, p. 476ff]), and the Levi component of $P_{\Pi \backslash\{\alpha\}}$ has again tiny weights. We get the statement by induction on the number of roots. Q.E.D.

Proposition 5.6 Theorem 5.4 holds for the spaces $G_{\mathbf{C}} / P_{\Theta_{j}}$ with $\Theta_{j}$ as in Lemma 5.9. In particular, it holds for $G_{\mathbf{C}} / B_{\mathbf{C}}$.

Proof: The proof proceeds by induction on the number of elements of $\Pi \backslash \Theta_{j}$. For $\Theta_{j}=\Pi$, the analytic torsion vanishes and the statement is trivial. Now assume that the statement is true for all $\Theta_{k} \supset \Theta_{j}$. To shorten the notation, we shall denote $K_{\Theta_{j}}$ and $K_{\Theta_{j+1}}$ by $H$ and $K$, respectively. Thus, the associated holomorphic fibration

$$
\pi: G_{c} / H \rightarrow G_{c} / K
$$

has as fiber the Hermitian symmetric space $K / H$. The Kähler metric on $G_{c} / H$ induces a Kähler metric on $K / H$. We fix a Kähler metric on $G_{c} / K$ associated to $X_{1} \in \mathfrak{t}_{c}$. Notice that $X_{1}$ and $X_{0}$ have to be different as their stabilizers are different.

By theorem 2.8 applied to $K / H$ and the base change formulae, we find that a certain shift of the direct image $R^{\bullet} \pi_{*} E_{\rho_{H}+\lambda}^{H}$ is given by the vector bundle $E_{\rho_{K}+\lambda}^{K}$ associated to the irreducible $K$-representation with highest weight $\lambda$. Also, the cohomologies $H^{\bullet}\left(G_{c} / H, E_{\rho_{H}+\lambda}^{H}\right)$ and $H^{\bullet}\left(G_{c} / K, E_{\rho_{K}+\lambda}^{K}\right)$ can both be identified with the irreducible $G$-representation $E_{\rho+\lambda}^{G}$, and all cohomology groups except one vanish. As a $G$-invariant metric on an irreducible representation is unique up to a constant, the isomorphism $\sigma$ in section 1 simply corresponds to a multiplication of the metric with a constant, and the equivariant metrics considered in theorem 4.1 differ just by a constant $C^{\prime} \in \mathbf{R}$ times $\chi_{\rho+\lambda}(t)$.

Let $\widetilde{\mathrm{Td}}_{t}\left(T G_{c} / H, T G_{c} / K, g_{X_{0}}, g_{X_{1}}\right)$ denote the equivariant Bott-Chern secondary class associated to the short exact sequence

$$
0 \rightarrow T K / H \rightarrow T G_{c} / H \rightarrow \pi^{*} T G_{c} / K \rightarrow 0
$$

of vector bundles on $G_{c} / H$, equipped with the Hermitian metrics induced by $X_{0}, X_{0}$ and $X_{1}$, respectively. By theorem 4.1 we get the formula

$$
\begin{aligned}
T_{t}\left(G_{c} / H, \bar{E}_{\rho_{H}+\lambda}^{H}\right)= & \log \|\sigma\|_{L^{2}, t}^{2}+T_{t}\left(G_{c} / K, \bar{E}_{\rho_{K}+\lambda}^{K}\right) \\
& +\int_{\left(G_{c} / K\right)^{t}} \operatorname{Td}_{t}\left(T G_{c} / K\right) T_{t}\left(K / H, \bar{E}_{\rho_{H}+\lambda}^{H}\right) \\
& -\int_{\left(G_{c} / H\right)^{t}} \widetilde{\mathrm{Td}_{t}}\left(T G_{c} / H, T G_{c} / K, g_{X_{0}}, g_{X_{1}}\right) \operatorname{ch}_{t}\left(E_{\rho_{H}+\lambda}^{H}\right)
\end{aligned}
$$

The fixed point set $\left(G_{c} / K\right)^{t}$ is given by $W_{G} / W_{K}$. For simplicity, we assume $\Psi^{-}=\emptyset$; the proof remains the same in the general case. 
By applying the induction hypothesis and [K2, Theorem 18] for the torsion of the fiber, we get

$$
\begin{aligned}
& T_{t}\left(G_{c} / H, \bar{E}_{\rho_{H}+\lambda}^{H}\right)=C^{\prime} \chi_{\rho+\lambda}(t)+\int_{\left(G_{c} / K\right)^{t}} \operatorname{Td}_{t}\left(T G_{c} / K\right) R_{t}\left(T G_{c} / K\right) \operatorname{ch}_{t}\left(E_{\rho_{K}+\lambda}^{K}\right) \\
& \quad-\sum_{\alpha \in \Sigma^{+} \backslash \Sigma_{K}^{+}} \sum_{k=1}^{\left\langle\alpha^{\vee}, \rho+\lambda\right\rangle} \chi_{\rho+\lambda-k \alpha}(t) \log k-\sum_{\alpha \in \Sigma^{+} \backslash \Sigma_{K}^{+}} \zeta \chi_{\rho+\lambda-k \alpha}(t) \log \alpha^{\vee}\left(X_{1}\right) \\
& +C^{\prime \prime} \chi_{\rho+\lambda}(t) \\
& +\sum_{w \in W_{G} / W_{K}} \operatorname{Td}_{t}\left(T G_{c} / K\right)\left(\left(\int_{(K / H)^{t}} \operatorname{Td}_{t}(T K / H) R_{t}(T K / H) \operatorname{ch}_{t}\left(E_{\rho_{H}+\lambda}^{H}\right)\right)_{\mid w K}\right. \\
& -\sum_{\alpha \in \Sigma_{K}^{+} \backslash \Sigma_{H}^{+}} \sum_{k=1}^{\left\langle\alpha^{\vee}, \rho_{K}+\lambda\right\rangle} \chi_{\rho_{K}+\lambda-k \alpha}^{K}(w t) \log k-\sum_{\alpha \in \Sigma_{K}^{+} \backslash \Sigma_{H}^{+}} \zeta \chi_{\rho_{K}+\lambda-k \alpha}^{K}(w t) \log \alpha^{\vee}\left(X_{0}\right) \\
& \left.-\chi_{\rho_{K}+\lambda}^{K}(t) \sum_{\alpha \in \Sigma_{K}^{+} \backslash \Sigma_{H}^{+}} \log \alpha^{\vee}\left(X_{0}\right)\right) \\
& -\int_{\left(G_{c} / H\right)^{t}} \widetilde{T_{t}}\left(T G_{c} / H, T G_{c} / K, g_{X_{0}}, g_{X_{1}}\right) \operatorname{ch}_{t}\left(\bar{E}_{\rho_{H}+\lambda}^{H}\right) .
\end{aligned}
$$

Next we notice that

$$
\begin{aligned}
& \int_{\left(G_{c} / K\right)^{t}} \operatorname{Td}_{t}\left(T G_{c} / K\right) R_{t}\left(T G_{c} / K\right) \operatorname{ch}_{t}\left(E_{\rho_{K}+\lambda}^{K}\right) \\
& +\int_{\left(G_{c} / K\right)^{t}} \operatorname{Td}_{t}\left(T G_{c} / K\right) \int_{(K / H)^{t}} \operatorname{Td}_{t}(T K / H) R_{t}(T K / H) \operatorname{ch}_{t}\left(E_{\rho_{H}+\lambda}^{H}\right) \\
= & \int_{\left(G_{c} / K\right)^{t}} \operatorname{Td}_{t}\left(T G_{c} / K\right) R_{t}\left(T G_{c} / K\right) \int_{(K / H)^{t}} \operatorname{Td}_{t}(T K / H) \operatorname{ch}_{t}\left(E_{\rho_{H}+\lambda}^{H}\right) \\
& +\int_{\left(G_{c} / H\right)^{t}} \operatorname{Td}_{t}\left(T G_{c} / H\right) R_{t}(T K / H) \operatorname{ch}_{t}\left(E_{\rho_{H}+\lambda}^{H}\right) \\
= & \int_{\left(G_{c} / H\right)^{t}} \operatorname{Td}_{t}\left(T G_{c} / H\right) R_{t}\left(T G_{c} / K\right) \operatorname{ch}_{t}\left(E_{\rho_{H}+\lambda}^{H}\right) \\
& +\int_{\left(G_{c} / H\right)^{t}} \operatorname{Td}_{t}\left(T G_{c} / H\right) R_{t}(T K / H) \operatorname{ch}_{t}\left(E_{\rho_{H}+\lambda}^{H}\right) \\
= & \int_{\left(G_{c} / H\right)^{t}} \operatorname{Td}_{t}\left(T G_{c} / H\right) R_{t}\left(T G_{c} / H\right) \operatorname{ch}_{t}\left(E_{\rho_{H}+\lambda}^{H}\right) .
\end{aligned}
$$

Also, by the classical Lefschetz fixed point formula (in this case already shown in Bott], see also [K2, Theorem 11, eq. (84)] and recall (2)) we get

$$
\sum_{w \in W_{G} / W_{K}} \operatorname{Td}_{t}\left(T G_{c} / K\right) \chi_{\rho_{K}+\lambda-k \alpha}^{K}(w t)=\chi_{\rho+\lambda-k \alpha}(t) ;
$$


furthermore, $\left\langle\alpha^{\vee}, \rho_{K}+\lambda\right\rangle=\left\langle\alpha^{\vee}, \rho+\lambda\right\rangle$ for $\alpha \in \Sigma_{K}$ by [K2, p. 106]. By the definition of $\widetilde{T d}$ we get

$$
\begin{aligned}
\int_{\left(G_{c} / H\right)^{t}} & \widetilde{\operatorname{Td}}_{t}\left(T G_{c} / H, T G_{c} / K, g_{X_{0}}, g_{X_{1}}\right) \operatorname{ch}_{t}\left(E_{\rho_{H}+\lambda}^{H}\right) \\
= & \sum_{w \in W_{G} / W_{H}} \frac{\chi_{\rho_{H}+\lambda}^{H}(w t)}{\prod_{\beta \in \Sigma^{+} \backslash \Sigma_{H}^{+}}\left(1-e^{-2 \pi i \beta(w t)}\right)} \sum_{\alpha \in \Sigma^{+} \backslash \Sigma_{K}^{+}} \frac{\log \alpha\left(X_{0}\right) / \alpha\left(X_{1}\right)}{e^{2 \pi i \alpha(w t)}-1} \\
= & \sum_{\alpha \in \Sigma^{+} \backslash \Sigma_{K}^{+}} \zeta \chi_{\rho+\lambda-k \alpha}(t) \log \frac{\alpha\left(X_{0}\right)}{\alpha\left(X_{1}\right)} .
\end{aligned}
$$

Thus, we find

$$
\begin{aligned}
T_{t}\left(G_{c} / H, \bar{E}_{\rho_{H}+\lambda}^{H}\right)=\left(C^{\prime}+C^{\prime \prime}\right) \chi_{\rho+\lambda}(t)+\int_{\left(G_{c} / H\right)^{t}} \operatorname{Td}_{t}\left(T G_{c} / H\right) R_{t}\left(T G_{c} / H\right) \mathrm{ch}_{t}\left(E_{\rho_{H}+\lambda}^{H}\right) \\
-\sum_{\alpha \in \Sigma^{+} \backslash \Sigma_{K}^{+}} \sum_{k=1}^{\left\langle\alpha^{\vee}, \rho+\lambda\right\rangle} \chi_{\rho+\lambda-k \alpha}(t) \log k-\sum_{\alpha \in \Sigma_{K}^{+} \backslash \Sigma_{H}^{+}} \sum_{k=1}^{\left\langle\alpha^{\vee}, \rho+\lambda\right\rangle} \chi_{\rho+\lambda-k \alpha}(t) \log k \\
-\sum_{\alpha \in \Sigma^{+} \backslash \Sigma_{K}^{+}} \zeta \chi_{\rho+\lambda-k \alpha}(t) \log \alpha^{\vee}\left(X_{1}\right)-\sum_{\alpha \in \Sigma_{K}^{+} \backslash \Sigma_{H}^{+}} \zeta \chi_{\rho+\lambda-k \alpha}(t) \log \alpha^{\vee}\left(X_{0}\right) \\
\quad-\sum_{\alpha \in \Sigma^{+} \backslash \Sigma_{K}^{+}} \boldsymbol{\zeta} \chi_{\rho+\lambda-k \alpha}(t) \log \frac{\alpha\left(X_{0}\right)}{\alpha\left(X_{1}\right)}-\chi_{\rho+\lambda}(t) \sum_{\alpha \in \Sigma_{K}^{+} \backslash \Sigma_{H}^{+}} \log \alpha^{\vee}\left(X_{0}\right) \\
=C \chi_{\rho+\lambda}(t)+\int_{\left(G_{c} / H\right)^{t}} \operatorname{Td}_{t}\left(T G_{c} / H\right) R_{t}\left(T G_{c} / H\right) \operatorname{ch}_{t}\left(E_{\rho_{H}+\lambda}^{H}\right) \\
\quad \sum_{\alpha \in \Sigma^{+} \backslash \Sigma_{H}^{+}} \sum_{k=1}^{\left\langle\alpha^{\vee}, \rho+\lambda\right\rangle} \chi_{\rho+\lambda-k \alpha}(t) \log k-\sum_{\alpha \in \Sigma^{+} \backslash \Sigma_{H}^{+}} \boldsymbol{\zeta} \chi_{\rho+\lambda-k \alpha}(t) \log \alpha^{\vee}\left(X_{0}\right) .
\end{aligned}
$$

\section{Q.E.D.}

Proof of Theorem 5.4: Consider the fibration $G_{c} / T_{c} \rightarrow G_{c} / K$ with fiber $K / T_{c}$. We equip $G_{c} / T_{c}$ by a Kähler metric associated to $X_{0} \in \mathfrak{t}_{c}$ with stabilizer $T_{c}$. Using again theorem 4.1 we get the formula

$$
\begin{aligned}
T_{t}\left(G_{c} / K, \bar{E}_{\rho_{K}+\lambda}^{K}\right)= & -\log |\sigma|_{L^{2}, t}^{2}+T_{t}\left(G_{c} / T_{c}, \bar{E}_{\lambda}^{T}\right) \\
& -\int_{\left(G_{c} / K\right)_{t}} \operatorname{Td}_{t}\left(T G_{c} / K\right) T_{t}\left(K / T_{c}, \bar{E}_{\lambda}^{T}\right) \\
& +\int_{\left(G_{c} / T\right)_{t}} \widetilde{\mathrm{Td}_{t}}\left(T G_{c} / T_{c}, T G_{c} / K, g_{X_{0}}, g_{X_{1}}\right) \operatorname{ch}_{t}\left(E_{\lambda}^{T}\right) .
\end{aligned}
$$

By applying the formulae for $T_{t}\left(G_{c} / T_{c}, \bar{E}_{\lambda}^{T}\right)$ and $T_{t}\left(K / T_{c}, \bar{E}_{\lambda}^{T}\right)$ shown in Proposition 5.6, we get the value of $T_{t}\left(G_{c} / K, \bar{E}_{\rho_{K}+\lambda}^{K}\right)$ by the analogue of the calculation above. Q.E.D. 


\section{Description of the $L^{2}$-metric}

Consider a vector bundle $E_{\rho_{K}+\lambda}^{K}$ on $M$ associated to a $\Sigma_{K^{-}}^{+}$-dominant weight $\lambda$. In this section we describe the $L^{2}$-metric on $H^{*}\left(M, E_{\rho_{K}+\lambda}^{K}\right)$ induced by embedding this space into the $C^{\infty}$ differential forms with coefficients in $E_{\rho_{K}+\lambda}^{K}$ via Hodge theory. As the cohomology is an irreducible $G$-representation, the metric is uniquely determined by the norm of one element.

Classically Bott, $\Gamma^{\infty}\left(G_{c} / K, E_{\rho_{K}+\lambda}^{K} \otimes \Lambda^{q} T^{* 0,1} G_{c} / K\right)$ has the $L^{2}$-dense subspace

$$
\bigoplus_{\mu \in I_{+}} \operatorname{Hom}_{K}\left(V_{\rho+\mu}, V_{\rho_{K}+\lambda}^{K} \otimes \Lambda^{q} \operatorname{Ad}_{G / P}^{1,0}\right) \otimes V_{\rho+\mu}
$$

where the section associated to $f \otimes v \in \operatorname{Hom}_{K}\left(V_{\rho+\mu}, V_{\rho_{K}+\lambda}^{K} \otimes \Lambda^{q} \operatorname{Ad}_{G / P}^{1,0}\right) \otimes V_{\rho+\mu}$ is given by the $K$-equivariant $C^{\infty}$-function

$$
\begin{aligned}
s: \quad G_{c} & \rightarrow V_{\rho_{K}+\lambda}^{K} \otimes \Lambda^{q} \operatorname{Ad}_{G / P}^{1,0} \\
g & \mapsto f\left(g^{-1} v\right) .
\end{aligned}
$$

The direct sum (12) is a direct sum of complexes. The summand for $\mu$ is the set of $K$-invariants in the tensor product of the complex $\operatorname{Hom}\left(V_{\rho+\mu}, \Lambda^{q} \mathrm{Ad}_{G / P}^{1,0}\right)$ with the space $V_{\rho_{K}+\lambda}^{K} \otimes V_{\rho+\mu}$. This last complex is canonically isomorphic to the standard complex calculating the Lie algebra cohomology for the nilpotent radical $\left[\mathrm{SB}\right.$, p. 89, eq. (***)], Bott, equation (15.3)]. Set $w$ such that $w^{-1}(\rho+$ $\lambda)=: \rho+\lambda_{0} \in I_{+}$. Then the cohomology has either highest weight $\lambda_{0}$ or it vanishes in case $\lambda_{0} \notin I_{+}$. Assume that $\lambda_{0} \in I_{+}$. According to the proof of Kostant's theorem V $\mathbf{V}$, Corollary 3.2.11] the first tensor factor of the summand associated to $\mu=\lambda_{0}$ in (12) consists only of a one-dimensional space in degree $l(w)$. Thus the cohomology is embedded uniquely in the complex (12) by the one-dimensional space

$$
\operatorname{Hom}_{K}\left(V_{\rho+\lambda_{0}}, V_{\rho_{K}+\lambda}^{K} \otimes \Lambda^{l(w)} \operatorname{Ad}_{G / P}^{1,0}\right) .
$$

Now assume that $V_{\rho_{K}+\lambda}^{K}$ is one-dimensional and equipped with an Hermitian metric. Fix one $v^{\prime} \in V_{\rho_{K}+\lambda}^{K} \otimes \Lambda^{l(w)} \operatorname{Ad}_{G / P}^{1,0}$ of weight $w \lambda_{0}$ and norm 1. Choose $v \in V_{\rho+\lambda_{0}}$ of weight $w \lambda_{0}$ and let $v^{\vee} \in V_{\rho+\lambda_{0}}^{\vee}$ be of weight $-w \lambda_{0}$ with $v^{\vee}(v)=1$. Then $v^{\vee} \otimes v^{\prime} \in \operatorname{Hom}_{K}\left(V_{\rho+\lambda_{0}}, V_{\rho_{K}+\lambda}^{K} \otimes \Lambda^{l(w)} \operatorname{Ad}_{G / P}^{1,0}\right)$, hence there is an element of $H^{l(w)}\left(G_{c} / K, E_{\rho_{K}+\lambda}^{K}\right)$ of weight $w \lambda_{0}$ given by the section

$$
s_{0}: g \mapsto v^{\vee}\left(g^{-1} v\right) \otimes v^{\prime} .
$$

which is independent of the choice of $v$.

Lemma 6.1 Let $M$ be equipped with the metric associated to $X_{0} \in \mathfrak{t}_{\mathrm{reg}}$. Then the $L^{2}$-norm of $s_{0}$ is given by

$$
\left|s_{0}\right|_{L^{2}}^{2}=\prod_{\alpha \in \Psi} \frac{\alpha^{\vee}\left(X_{0}\right)}{\left\langle\alpha^{\vee}, \rho+\lambda\right\rangle} .
$$


Proof: According to $\mathrm{BeGeV}$, Lemma 7.23], the computation reduces to that for the measure $d \bar{g}$ on $\bar{M}$ induced by the Haar measure $d g$ via the formula

$$
\left|s_{0}\right|_{L^{2}}^{2}=\prod_{\alpha \in \Psi} \frac{\alpha^{\vee}\left(X_{0}\right)}{\left\langle\alpha^{\vee}, \rho\right\rangle} \int_{G_{c} / K}\left|s_{0}(\bar{g})\right|^{2} d \bar{g}
$$

(by [K2, (71)], our normalized Haar measure differs from the measure considered in BeGeV, Lemma 7.23] by the factor in the denominator). By the $K$-invariance of $\left|s_{0}\right|^{2}$, this equals

$$
\begin{aligned}
\left|s_{0}\right|_{L^{2}}^{2} & =\prod_{\alpha \in \Psi} \frac{\alpha^{\vee}\left(X_{0}\right)}{\left\langle\alpha^{\vee}, \rho\right\rangle} \cdot \int_{G}\left|v^{\vee}\left(g^{-1} v\right)\right|^{2} d g \\
& =\prod_{\alpha \in \Psi} \frac{\alpha^{\vee}\left(X_{0}\right)}{\left\langle\alpha^{\vee}, \rho\right\rangle} \cdot\left(\operatorname{dim} V_{\rho+\lambda}\right)^{-1} .
\end{aligned}
$$

The last equality follows by the orthogonality relations ( $\mathrm{BtD}$, Th. 4.5]). As $\operatorname{dim} V_{\rho_{k}+\lambda}^{K}=1$, the Weyl dimension formula shows

$$
\operatorname{dim} V_{\rho+\lambda}=\prod_{\alpha \in \Sigma^{+}} \frac{\left\langle\alpha^{\vee}, \rho+\lambda\right\rangle}{\left\langle\alpha^{\vee}, \rho\right\rangle}=\prod_{\alpha \in \Psi} \frac{\left\langle\alpha^{\vee}, \rho+\lambda\right\rangle}{\left\langle\alpha^{\vee}, \rho\right\rangle} .
$$

Thus the Lemma follows. Q.E.D.

\section{The main result}

Theorem 7.1 Let $G$ be a Chevalley group having tiny weights and let $P$ be a standard parabolic subgroup. Consider a P-module A, free of rank one over $\mathbf{Z}$, equipped with an Hermitian metric on $A_{\mathbf{C}}$. Let $\lambda$ be the weight of $A$ and choose $w \in W_{G}$ such that $w^{-1}(\rho+\lambda)=\rho+\lambda_{0} \in I_{+}$. We denote the induced line bundle on the $n+1$-dimensional variety $X=G / P$ by $\mathcal{L}_{\lambda}$. There is a constant $C \in \mathbf{R}$ such that the following identity of formal linear combinations of weights holds

$$
\begin{aligned}
-(-1)^{l(w)} \sum_{\mu \in X^{*}(T)} \mu \log \operatorname{covol} \overline{H^{l(w)}\left(X, \mathcal{L}_{\lambda}\right)_{\mu}} \\
+\sum_{q=0}^{n}(-1)^{q} \sum_{\mu \in X^{*}(T)} \mu \log \# H^{q}\left(X, \mathcal{L}_{\lambda}\right)_{\mu, \text { tor }} \\
=-\frac{1}{2} \sum_{\alpha \in \Psi^{+}} \sum_{k=1}^{\left\langle\alpha^{\vee}, \rho+\lambda\right\rangle} \chi_{\rho+\lambda-k \alpha} \cdot \log k+\frac{1}{2} \sum_{\alpha \in \Psi^{-}} \sum_{k=1}^{\left\langle-\alpha^{\vee}, \rho+\lambda\right\rangle} \chi_{\rho+\lambda+k \alpha} \cdot \log k \\
\quad+(C-\log \operatorname{covol} \bar{A}) \chi_{\rho+\lambda} .
\end{aligned}
$$

Furthermore,

$$
C=-\frac{1}{2} \sum_{\alpha \in \Psi^{+}} \log \alpha^{\vee}\left(X_{0}\right)
$$


if either $G_{c} / K$ is Hermitian symmetric or if $\lambda \in I_{+}$.

Proof: Since $T(\mathbf{C})_{\operatorname{Reg}}:=\left\{t \in T(\mathbf{C})_{\text {tor }} \mid \alpha(t) \neq \beta(t) \forall \alpha, \beta \in \Sigma, \alpha \neq \beta\right\}$ is Zariski-dense in $T(\mathbf{C})$, it is enough to prove the identities of complex numbers obtained by substituting $\mu$ with $\mu(t) \in S^{1}$ for all $t \in T(\mathbf{C})_{\text {Reg. We apply [KR2, }}$ Th. 6.14] for imbeddings $\mu_{N} \hookrightarrow T$ satisfying property (Reg) (see definition 2.4) and for $t \in \mu_{N}(\mathbf{C})$ being a generator. We adopt the notations from [KR2, section 4].

Classically, the free part of the cohomology $H^{q}\left(X, \mathcal{L}_{\lambda}\right)$ vanishes in all degrees except at most for $q=l(w)$ Bott]. According to KR2, Th. 6.14],

$$
\begin{aligned}
-(-1)^{l(w)} \sum_{\mu \in X^{*}\left(\mu_{N}\right)} \mu(t) \log \operatorname{covol} \overline{H^{l(w)}\left(X, \mathcal{L}_{\lambda}\right)_{\mu}} \\
\quad+\sum_{q=0}^{n}(-1)^{q} \sum_{\mu \in X^{*}\left(\mu_{N}\right)} \mu(t) \log \# H^{q}\left(X, \mathcal{L}_{\lambda}\right)_{\mu, \text { tor }} \\
=\frac{1}{2} T_{t}\left(X_{\mathbf{C}}, \bar{L}_{\lambda, \mathbf{C}}\right)-\frac{1}{2} \int_{X(\mathbf{C})_{t}} \operatorname{Td}_{t}\left(\overline{T X}_{\mathbf{C}}\right) R_{t}\left(\overline{T X}_{\mathbf{C}}\right) \operatorname{ch}_{t}\left(\bar{L}_{\lambda}\right) \\
+\widehat{\operatorname{deg}} f_{*}\left(\widehat{\mathrm{Td}}_{\mu_{N}}(\overline{T f}) \widehat{\operatorname{ch}}_{\mu_{N}}\left(\bar{L}_{\lambda}\right)\right) .
\end{aligned}
$$

Let $f: X \rightarrow$ Spec $\mathbf{Z}$ be the structure morphism. As $X^{\mu_{N}}=X^{T}, T$ acts on $T f_{\mid X^{\mu_{N}}}$. Let $T f_{\alpha}$ denote the direct summand of $T f_{\mid X^{\mu_{N}}}$ associated to a weight $\alpha$. By Corollary 2.3, the components of the fixed point scheme are indexed by $W_{G} / W_{P}$. When restricted to the component $X_{w}^{\mu_{N}}$ for $[w] \in W_{G} / W_{P}$, the equivariant characteristic classes are given by

$$
\widehat{\operatorname{Td}}_{\mu_{N}}(\overline{T f})_{\mid X_{w}^{\mu_{N}}}=\prod_{\alpha \in \Psi}\left(1-e^{-2 \pi i w \alpha(t)}\right)^{-1} \cdot\left(1+\sum_{\alpha \in \Psi} \frac{\hat{c}_{1}\left(\overline{T f}_{w \alpha}\right)}{1-e^{2 \pi i w \alpha(t)}}\right)
$$

(compare the expansion of the equivariant Todd-class in [KR2, section 3.3]) and

$$
\widehat{c h}_{\mu_{N}}\left(\overline{\mathcal{L}}_{\lambda}\right)_{\mid X_{w}^{\mu_{N}}}=e^{2 \pi i w \lambda(t)}\left(1+\hat{c}_{1}\left(\overline{\mathcal{L}}_{\lambda}\right)\right) .
$$

Hence we get by Lemma 2.7

$$
\begin{aligned}
& \widehat{\operatorname{deg}} f_{*}\left(\widehat{\operatorname{Td}}_{\mu_{N}}(\overline{T f}) \widehat{\operatorname{ch}}_{\mu_{N}}\left(\overline{\mathcal{L}}_{\lambda}\right)\right) \\
& =\sum_{[w] \in W_{G} / W_{P}} \frac{e^{2 \pi i w \lambda(t)}}{\prod_{\alpha \in \Psi}\left(1-e^{-2 \pi i w \alpha(t)}\right)}\left[\sum_{\alpha \in \Psi} \frac{\widehat{\operatorname{deg}} f_{*} \hat{c}_{1}\left(\overline{T f}_{w \alpha}\right)}{1-e^{2 \pi i w \alpha(t)}}+\widehat{\operatorname{deg}} f_{*} \hat{c}_{1}\left(\overline{\mathcal{L}}_{\lambda}\right)\right] \\
& =\sum_{[w] \in W_{G} / W_{P}} \frac{e^{2 \pi i w \lambda(t)}}{\prod_{\alpha \in \Psi}\left(1-e^{-2 \pi i w \alpha(t)}\right)}\left[\frac{1}{2} \sum_{\alpha \in \Psi} \frac{-\log \alpha^{\vee}\left(X_{0}\right)}{1-e^{2 \pi i w \alpha(t)}}-\log \operatorname{covol} \bar{A}\right]
\end{aligned}
$$

Furthermore, [K2, Th. 11, eq. (84)] shows

$$
\sum_{[w] \in W_{G} / W_{P}} \frac{-e^{2 \pi i w \lambda(t)} \log \operatorname{covol} \bar{A}}{\prod_{\alpha \in \Psi}\left(1-e^{-2 \pi i w \alpha(t)}\right)}=-\chi_{\rho+\lambda}(t) \cdot \log \operatorname{covol} \bar{A}
$$


Similarly we shall proceed as in the proof of [K2 theorem 10] to obtain a formula for the other term. For a class $[w] \in W_{G} / W_{P}$ we shall denote by $l(w)$ the minimal length of its representents.

$$
\begin{aligned}
& \sum_{[w] \in W_{G} / W_{P}} \frac{e^{2 \pi i w \lambda(t)}}{\prod_{\alpha \in \Psi}\left(1-e^{-2 \pi i w \alpha(t)}\right)} \sum_{\alpha \in \Psi} \frac{-\log \alpha^{\vee}\left(X_{0}\right)}{1-e^{2 \pi i w \alpha(t)}} \\
= & \sum_{[w] \in W_{G} / W_{P}} \frac{e^{2 \pi i w\left(\rho-\rho_{K}+\lambda\right)(t)}(-1)^{l(w)} \sum_{w^{\prime} \in W_{P}} e^{2 \pi i w w^{\prime} \rho_{K}(t)}}{\prod_{\beta \in \Sigma^{+}} 2 i \sin \pi \beta(t)} \sum_{\alpha \in \Psi} \frac{\log \alpha^{\vee}\left(X_{0}\right)}{e^{2 \pi i w \alpha(t)}-1} \\
= & \sum_{[w] \in W_{G} / W_{P}} \frac{1}{\prod_{\beta \in \Sigma^{+}} 2 i \sin \pi \beta(t)} \\
& \cdot \sum_{\alpha \in \Psi} \sum_{w^{\prime} \in W_{P}}(-1)^{l(w)+l\left(w^{\prime}\right)} e^{2 \pi i w w^{\prime}(\rho+\lambda)(t)} \frac{\log \left(w^{\prime} \alpha\right)^{\vee}\left(X_{0}\right)}{e^{2 \pi i w w^{\prime} \alpha(t)}-1}
\end{aligned}
$$

(in the last equation we used the facts that $\lambda$ and $\Psi$ are $W_{P}$-invariant)

$$
=\sum_{\alpha \in \Psi} \sum_{w \in W_{G}} \frac{(-1)^{l(w)} e^{2 \pi i w(\rho+\lambda)(t)}\left(e^{2 \pi i w \alpha(t)}-1\right)^{-1}}{\prod_{\beta \in \Sigma^{+}} 2 i \sin \pi \beta(t)} \log \alpha^{\vee}\left(X_{0}\right)
$$

(as $X_{0}$ is $K$-stable)

$$
=\sum_{\alpha \in \Psi} \zeta \chi_{\rho+\lambda-k \alpha}(t) \cdot \log \alpha^{\vee}\left(X_{0}\right)
$$

(by equation (11)). Thus

$$
\begin{aligned}
& \widehat{\operatorname{deg}} f_{*}\left(\widehat{\operatorname{Td}}_{\mu_{N}}(\overline{T f}) \widehat{\operatorname{ch}}_{\mu_{N}}\left(\overline{\mathcal{L}}_{\lambda}\right)\right) \\
& \quad=\frac{1}{2} \sum_{\alpha \in \Psi} \zeta \chi_{\rho+\lambda-k \alpha}(t) \cdot \log \alpha^{\vee}\left(X_{0}\right)-\chi_{\rho+\lambda}(t) \cdot \log \operatorname{covol} \bar{A} .
\end{aligned}
$$

The proof is finished by combining this result with Th. 5.4. For Hermitian symmetric spaces, combine instead with the more precise corollary 5.3 which does not involve the unknown constant $C$. Now consider the case $\lambda \in I_{+}$. In this case, by the Kempf vanishing theorem [J2, Prop. 4.5] the cohomology has no torsion. Furthermore $\Psi=\Psi^{+}$, thus the formula in theorem 7.1 simplifies to

$$
\begin{aligned}
& -\sum_{\mu \in X^{*}(T)} \mu \log \operatorname{covol} \overline{H^{0}\left(X, \mathcal{L}_{\lambda}\right)_{\mu}} \\
& =-\frac{1}{2} \sum_{\alpha \in \Psi} \sum_{k=1}^{\left\langle\alpha^{\vee}, \rho+\lambda\right\rangle} \chi_{\rho+\lambda-k \alpha} \cdot \log k+(C-\log \operatorname{covol} \bar{A}) \chi_{\rho+\lambda} .
\end{aligned}
$$

In particular, the component associated to $\mu=\lambda$ verifies the equality

$$
-\log \operatorname{covol} \overline{H^{0}\left(X, \mathcal{L}_{\lambda}\right)}{ }_{\lambda}=\frac{1}{2} \sum_{\alpha \in \Psi} \log \left\langle\alpha^{\vee}, \rho+\lambda\right\rangle+(C-\log \operatorname{covol} \bar{A}),
$$


as the weight $\lambda$ occurs only in the characters $\chi_{\rho+\lambda}$ and $\chi_{\rho+\lambda-\left\langle\alpha^{\vee}, \rho+\lambda\right\rangle \alpha}$, with multiplicity 1 and -1 , respectively. By combining Proposition 2.12 and Lemma 6.1. we find on the other hand

$-\log \operatorname{covol}{\overline{H^{0}\left(X, \mathcal{L}_{\lambda}\right)_{\lambda}}}=-\frac{1}{2} \sum_{\alpha \in \Psi} \log \alpha^{\vee}\left(X_{0}\right)+\frac{1}{2} \sum_{\alpha \in \Psi} \log \left\langle\alpha^{\vee}, \rho+\lambda\right\rangle-\log \operatorname{covol} \bar{A}$

(in Lemma 6.1, $\bar{A}$ was normalized to have covolume 1 ). Thus we get the value of the constant $C$ for dominant $\lambda$. Q.E.D.

Corollary 7.2 Let $A_{\rho+\lambda}:=H^{l(w)}\left(X, \mathcal{L}_{\lambda}\right)_{\text {free }}$ be a $G$-module induced by a line bundle $\mathcal{L}_{\lambda}$. If $A_{\rho+\lambda} \neq 0$ (i.e. $\lambda_{0} \in I_{+}$) equip $A_{\rho+\lambda}$ with the unique $G_{c}$-invariant metric such that the generator of the weight space to the highest weight $\lambda_{0}$ has norm 1. Then

$$
\begin{aligned}
& -(-1)^{l(w)} \sum_{\mu \in X^{*}(T)} \mu \log \operatorname{covol} \bar{A}_{\rho+\lambda, \mu} \\
& \quad+\sum_{q=0}^{n}(-1)^{q}\left(\sum_{\mu \in X^{*}(T)} \mu \log \# H^{q}\left(X, \mathcal{L}_{\lambda}\right)_{\mu, \text { tor }}-(-1)^{l(w)} \chi_{\rho+\lambda} \log \# H^{q}\left(X, \mathcal{L}_{\lambda}\right)_{\lambda_{0}, \text { tor }}\right) \\
& =-\frac{1}{2} \sum_{\alpha \in \Psi^{+}} \sum_{k=1}^{\left\langle\alpha^{\vee}, \rho+\lambda\right\rangle-1} \chi_{\rho+\lambda-k \alpha} \cdot \log k+\frac{1}{2} \sum_{\alpha \in \Psi^{-}} \sum_{k=1}^{\left\langle-\alpha^{\vee}, \rho+\lambda\right\rangle-1} \chi_{\rho+\lambda+k \alpha} \cdot \log k
\end{aligned}
$$

Proof: This follows from theorem 7.1 by comparing the components of weight $\lambda_{0}$. The weight $\lambda_{0}$ occurs only in the characters $\chi_{\rho+\lambda}$ and $\chi_{\rho+\lambda-\left\langle\alpha^{\vee}, \rho+\lambda\right\rangle \alpha}$, with multiplicity $(-1)^{l(w)}$ and $-(-1)^{l(w)}$, respectively. Q.E.D.

Remark. Let $P^{\prime} \subseteq P$ be another standard parabolic subgroup. Then $A$ is a $P^{\prime}$-representation, and both sides of the above formula remain the same. Namely, $H^{\bullet}\left(G / P, \mathcal{L}_{\lambda}\right)=H^{\bullet}\left(G / P^{\prime}, \mathcal{L}_{\lambda}\right)$ and $\chi_{\rho+\lambda-k \alpha}=0$ for any $\alpha \in \Sigma_{K}^{+}$, $1 \leq k \leq\left\langle\alpha^{\vee}, \rho+\lambda\right\rangle-1$.

For two $\mathbf{Z}$-free $G$-modules $A, A^{\prime}$ with $A_{\mathbf{Q}}$ and $A_{\mathbf{Q}}^{\prime}$ isomorphic and irreducible we denote by $\left[A, A^{\prime}\right] \in \mathbf{Q}^{+}$the index obtained by embedding $A^{\prime}$ in such a way in $A \otimes \mathbf{Q}$ that the weight spaces of highest weight are identified. Then $\left[A, A^{\prime}\right]=\operatorname{covol} A^{\prime} / \operatorname{covol} A$. Let $w_{0}$ be the Weyl group element of maximal length. When applied to $w_{0} \cdot \lambda$ instead of $\lambda$, the above corollary reads

$$
\begin{aligned}
& -\frac{1}{2} \sum_{\alpha \in \Psi^{+}} \sum_{k=1}^{\left\langle\alpha^{\vee}, \rho+\lambda\right\rangle-1} \chi_{\rho+\lambda-k \alpha} \cdot \log k+\frac{1}{2} \sum_{\alpha \in \Psi^{-}} \sum_{k=1}^{\left\langle-\alpha^{\vee}, \rho+\lambda\right\rangle-1} \chi_{\rho+\lambda+k \alpha} \cdot \log k \\
& =-\frac{(-1)^{l\left(w_{0}\right)}}{2} \sum_{\alpha \in \Psi^{+}} \sum_{k=1}^{-\left\langle-w_{0} \alpha^{\vee}, w_{0}(\rho+\lambda)\right\rangle-1} \chi_{w_{0}(\rho+\lambda-k \alpha)} \cdot \log k \\
& +\frac{(-1)^{l\left(w_{0}\right)}}{2} \sum_{\alpha \in \Psi^{-}} \sum_{k=1}^{\left\langle-w_{0} \alpha^{\vee}, w_{0}(\rho+\lambda)\right\rangle-1} \chi_{w_{0}(\rho+\lambda+k \alpha)} \cdot \log k
\end{aligned}
$$




$$
\begin{aligned}
= & -\frac{(-1)^{l\left(w_{0}\right)}}{2} \sum_{\alpha \in-w_{0} \Psi^{+}} \sum_{k=1}^{-\left\langle\alpha^{\vee}, w_{0}(\rho+\lambda)\right\rangle-1} \chi_{w_{0}(\rho+\lambda)+k \alpha} \cdot \log k \\
& +\frac{(-1)^{l\left(w_{0}\right)}}{2} \sum_{\alpha \in-w_{0} \Psi^{-}} \sum_{k=1}^{\left\langle\alpha^{\vee}, w_{0}(\rho+\lambda)\right\rangle-1} \chi_{w_{0}(\rho+\lambda)-k \alpha} \cdot \log k \\
= & (-1)^{l(w)} \sum_{\mu \in X^{*}(T)} \mu \log \operatorname{covol} \bar{A}_{w_{0}(\rho+\lambda), \mu} \\
& -\sum_{q=0}^{n}(-1)^{q+l\left(w_{0}\right)}\left(\sum_{\mu \in X^{*}(T)} \mu \log \# H^{q}\left(X, \mathcal{L}_{w_{0} . \lambda}\right)_{\mu, \text { tor }}\right. \\
& \left.-(-1)^{l\left(w_{0} w\right)} \chi_{w_{0}(\rho+\lambda)} \log \# H^{q}\left(X, \mathcal{L}_{w_{0} . \lambda}\right)_{\lambda_{0}, \text { tor }}\right) \\
= & (-1)^{l(w)} \sum_{\mu \in X^{*}(T)} \mu \log \operatorname{covol} \bar{A}_{w_{0}(\rho+\lambda), \mu} \\
& -\sum_{q=0}^{n}(-1)^{q}\left(\sum_{\mu \in X^{*}(T)} \mu \log \# H^{n-q}\left(X, \mathcal{L}_{w_{0} . \lambda}\right)_{\mu, \text { tor }}\right. \\
& \left.-(-1)^{l(w)} \chi_{\rho+\lambda} \log \# H^{n-q}\left(X, \mathcal{L}_{w_{0} . \lambda}\right)_{\lambda_{0}, \text { tor }}\right) .
\end{aligned}
$$

By subtracting the equations for $A_{\rho+\lambda}$ and $A_{w_{0}(\rho+\lambda)}$ we get an identity which is an immediate consequence of Corollary 2.10 and Proposition 2.11. Thus corollary 7.2 is equivalent to the statement obtained by adding the equations for $A_{\rho+\lambda}$ and $A_{w_{0}(\rho+\lambda)}$. We get

Corollary 7.3 (Jantzen sum formula [Ja, $p .311]$ )

$$
\begin{aligned}
(-1)^{l(w)} & \sum_{\mu \in X^{*}(T)} \mu \log \left[A_{\rho+\lambda, \mu}: A_{w_{0}(\rho+\lambda), \mu}\right] \\
& +\sum_{\mu \in X^{*}(T)} \sum_{q=0}^{n} \mu \cdot(-1)^{q}\left(\log \# H^{q}\left(X, \mathcal{L}_{\lambda}\right)_{\mu, \text { tor }}-\log \# H^{n-q}\left(X, \mathcal{L}_{w_{0} \cdot \lambda}\right)_{\mu, \text { tor }}\right) \\
& -(-1)^{l(w)} \chi_{\rho+\lambda} \sum_{q=0}^{n}(-1)^{q}\left(\log \# H^{q}\left(X, \mathcal{L}_{\lambda}\right)_{\lambda_{0}, \text { tor }}-\log \# H^{n-q}\left(X, \mathcal{L}_{w_{0}, \lambda}\right)_{\lambda_{0}, \text { tor }}\right) \\
= & -\sum_{\alpha \in \Psi^{+}} \sum_{k=1}^{\left\langle\alpha^{\vee}, \rho+\lambda\right\rangle-1} \chi_{\rho+\lambda-k \alpha} \cdot \log k+\sum_{\alpha \in \Psi^{-}} \sum_{k=1}^{\left\langle-\alpha^{\vee}, \rho+\lambda\right\rangle-1} \chi_{\rho+\lambda+k \alpha} \cdot \log k .
\end{aligned}
$$

As above, both sides are independent of the parabolic subgroup. In particular, for $P=B$ we get the usual formulation of the Jantzen sum formula.

Combining these formulae with $\mathrm{J} 2$, (8.7)]

$$
\# H^{q}\left(X, \mathcal{L}_{\lambda}\right)_{\lambda_{0}, \text { tor }}=0 \quad \forall \lambda, q
$$


one notices the vanishing of the multiples of $\chi_{\rho+\lambda}$ in the above two corollaries. We did not use this result before to point out what exactly our result says without applying further representation theory. For $\lambda \in I_{+}$, the Kempf vanishing theorem [J2, Prop. 4.5] states that the torsion of the cohomology vanishes. Note that the other way round the Jantzen sum formula provides the values of the equivariant Ray-Singer torsion for isolated fixed points up to a multiple of $\chi_{\rho+\lambda}$, in particular for the types $G_{2}, F_{4}$ and $E_{8}$.

\section{The height of generalized flag varieties}

For the definitions of the objects in Arakelov geometry which are used in this chapter we refer the reader to [SABK]. According to [BB], 14.7], the very ample line bundles on $G_{c} / K$ are induced by the $K$-representations with highest weight $\lambda$ such that

$$
\left\langle\alpha^{\vee}, \lambda\right\rangle= \begin{cases}=0 & \text { if } \alpha \in \Sigma_{K} \\ >0 & \text { if } \alpha \in \Psi\end{cases}
$$

Equip a line bundle $\mathcal{L}_{\lambda} \rightarrow G / P$ to such a $\lambda$ with the equivariant metric induced by normalizing the length of the generator of the $P$-module to 1 . Thus, as the equivariant metric on $\mathcal{L}_{\lambda}$ is unique up to a factor, it coincides with the metric induced by the $\mathcal{O}(1)$ bundle via the projective embedding associated to $\mathcal{L}_{\lambda}$. In particular, this metric is positive (this can be shown directly, too). In this section we compute the global height of $G / P$ with respect to $\overline{\mathcal{L}}_{\lambda}$ defined as

$$
h\left(G / P, \overline{\mathcal{L}}_{\lambda}\right):=f_{*}\left[\hat{c}_{1}\left(\overline{\mathcal{L}}_{\lambda}\right)^{n+1}\right] .
$$

Remark. The line bundle $\mathcal{L}_{\lambda}$ is also very ample [J2, II.8.5] (we shall not need this fact). See [A], Abbes] for relations between the global height of a variety and the height of points on that variety in this case.

Set $\Psi_{j}:=\left\{\alpha \in \Psi \mid\left\langle\alpha^{\vee}, \lambda\right\rangle=j\right\}$ for $j \in \mathbf{N}$. These sets are $W_{K}$-invariant as $\Psi$ and $\lambda$ are $W_{K}$-invariant. Hence for every $j$ there is a virtual $P$-representation with character equal to $\sum_{\alpha \in \Psi_{j}} e^{2 \pi i \alpha}$, defining a virtual vector bundle $E_{j}$ on $G / P$. As before, we equip $G_{c} / K$ with the metric associated to some $X_{0}$ in a certain subset of $\mathfrak{t}_{c}$.

Let $\mathrm{Ht}$ denote the additive topological characteristic class associated to the power series

$$
\operatorname{Ht}(x):=\sum_{k=0}^{\infty} \frac{(-x)^{k}}{2(k+1)(k+1) !} .
$$

This is the Taylor expansion of the function $x \mapsto \frac{1}{2 x}\left(\log |x|-\Gamma^{\prime}(1)-\operatorname{Ei}(-x)\right)$ at $x=0$ with Ei being the exponential-integral function $\mathbb{N}, 39$ (13)]. For $x<0$ $\operatorname{Ei}(x)$ is given by

$$
\operatorname{Ei}(x)=\int_{-\infty}^{x} \frac{e^{t}}{t} d t
$$


Theorem 8.1 The height of $G / P$ with respect to $\overline{\mathcal{L}}_{\lambda}$ is given by

$$
h\left(G / P, \overline{\mathcal{L}}_{\lambda}\right)=(n+1) ! \int_{M} \operatorname{Ht}\left(\sum_{j \in \mathbf{N}} j \psi^{j} E_{j}\right) e^{c_{1}\left(L_{\lambda}\right)}
$$

where $\psi^{j}$ denotes the $j$-th Adams operator.

In particular the height can be written as

$$
\sum_{l=0}^{n} \frac{(-1)^{l}}{2(l+1)}\left(\begin{array}{c}
n+1 \\
l+1
\end{array}\right) \int_{M} l ! \sum_{j \in \mathbf{N}} j^{l+1} \operatorname{ch}\left(E_{j}\right)^{[l]} c_{1}\left(L_{\lambda}\right)^{n-l} .
$$

We conclude that $h\left(G / P, \overline{\mathcal{L}}_{\lambda}\right)$ has the form

$$
\sum_{\ell=1}^{n+1} \frac{k_{\ell}}{2 \ell}
$$

with $k_{\ell} \in \mathbf{Z} \forall \ell$. See Corollary 8.2 for a refinement and a conjecture.

Proof: As in SABK, VIII.2.3] we have for $m \rightarrow \infty$

$$
r(m):=\widehat{\operatorname{deg}}\left(\pi_{*} \overline{\mathcal{L}}_{m \lambda},\|\cdot\|_{L^{2}}^{2}\right)=\frac{m^{n+1}}{(n+1) !} h\left(G / P, \overline{\mathcal{L}}_{\lambda}\right)+O\left(m^{n} \log m\right) .
$$

Here we use the fact that $\overline{\mathcal{L}}_{\lambda}$ is positive, which implies a result by BismutVasserot [BV] on the asymptotics of the non-equivariant holomorphic torsion. Consider the polynomials $d_{\rho+m \lambda-k \alpha}:=\operatorname{dim} V_{\rho+m \lambda-k \alpha}(\alpha \in \Psi)$ in $m$ and $k$, given by the Weyl dimension formula

$$
d_{\rho+m \lambda-k \alpha}=\prod_{\beta \in \Sigma^{+}}\left(1+\frac{\left\langle\beta^{\vee}, m \lambda-k \alpha\right\rangle}{\left\langle\beta^{\vee}, \rho\right\rangle}\right) .
$$

The term $r(m)$ equals the left hand side of theorem 7.1 evaluated at zero. By the Jantzen sum formula (or theorem 7.1 for $G$ having tiny weights), for $m \geq 1$

$$
\begin{aligned}
r(m)= & -\frac{1}{2} \sum_{\alpha \in \Psi} \sum_{k=1}^{\left\langle\alpha^{\vee}, \rho+m \lambda\right\rangle} d_{\rho+m \lambda-k \alpha} \log k \\
& -d_{\rho+m \lambda} \cdot\left[\log \operatorname{covol}{\overline{H^{0}\left(X, \mathcal{L}_{m \lambda}\right)}}_{m \lambda}+\frac{1}{2} \sum_{\alpha \in \Psi} \log \left\langle\alpha^{\vee}, \rho+m \lambda\right\rangle\right] .
\end{aligned}
$$

Here we replaced the constant $C$ in theorem 7.1 by the value one obtains by comparing the components of weight $m \lambda$, similar to the last section in the proof of theorem 7.1. As in the case $\lambda \in I_{+}$in theorem 7.1 (where only groups $G$ with tiny weights were allowed), we find again by combining Proposition 2.12 and Lemma 6.1

$$
\log \operatorname{covol}{\overline{H^{0}\left(X, \mathcal{L}_{m \lambda}\right)}}_{m \lambda}+\frac{1}{2} \sum_{\alpha \in \Psi} \log \left\langle\alpha^{\vee}, \rho+m \lambda\right\rangle=\frac{1}{2} \sum_{\alpha \in \Psi} \log \alpha^{\vee}\left(X_{0}\right),
$$


thus this factor is independent of $m$. The expression for $r(m)$ is a sum over terms which (at a first sight) look like having order $O\left(m^{1+\# \Sigma^{+}} \log m\right)$ for $m \rightarrow \infty$. Thus, it is not obvious that it is in fact of order $O\left(m^{n+1}\right)$, and one has to be careful when calculating the term of highest degree. We shall need the following three facts about the polynomials $d_{\rho+m \lambda-k \alpha}$ :

1. The degree in $m$ of $d_{\rho+m \lambda-k \alpha}$ is equal to $n$, since

$$
d_{\rho+m \lambda-k \alpha}=\prod_{\beta \in \Psi} \frac{\left\langle\beta^{\vee}, \rho+m \lambda-k \alpha\right\rangle}{\left\langle\beta^{\vee}, \rho\right\rangle} \cdot \prod_{\beta \in \Sigma_{K}^{+}} \frac{\left\langle\beta^{\vee}, \rho-k \alpha\right\rangle}{\left\langle\beta^{\vee}, \rho\right\rangle} .
$$

2. For all $j \in \mathbf{N}$, the common polynomial degree in $m$ and $k$ of $\sum_{\alpha \in \Psi_{j}} d_{\rho+m \lambda-k \alpha}$ is less or equal to $n$. This is a consequence of the Riemann-Roch theorem, which states in this case

$$
\sum_{\alpha \in \Psi_{j}} d_{\rho+m \lambda-k \alpha}=\int_{M} \operatorname{Td}(T M) \operatorname{ch}\left(\psi^{-k} E_{j}\right) e^{m c_{1}\left(L_{\lambda}\right)}
$$

(compare [K2, Th. 13]).

3. The polynomial $d_{\rho+m \lambda-k \alpha}$ is skew-symmetric in $k$ around $k=\left\langle\alpha^{\vee}, \rho+\right.$ $m \lambda\rangle / 2$. More precisely, by applying the reflection $S_{\alpha}$ at the hyperplane orthogonal to $\alpha$ we get

$$
d_{\rho+m \lambda-\left(k+\left\langle\alpha^{\vee}, \rho+m \lambda\right\rangle\right) \alpha}=-d_{\rho+m \lambda+k \alpha} .
$$

Now we see that

$$
\begin{aligned}
-2 r(m)+O\left(m^{n}\right)= & \sum_{j} \sum_{\alpha \in \Psi_{j}} \sum_{k=1}^{j m} d_{\rho+m \lambda-k \alpha} \log k \\
& +\sum_{\alpha \in \Psi} \sum_{k=\left\langle\alpha^{\vee}, m \lambda\right\rangle+1}^{\left\langle\alpha^{\vee}, \rho+m \lambda\right\rangle} d_{\rho+m \lambda-k \alpha} \log k \\
= & \sum_{j} \sum_{k=1}^{j m} \sum_{\alpha \in \Psi_{j}} d_{\rho+m \lambda-k \alpha} \log k \\
& -\sum_{\alpha \in \Psi} \sum_{k=1-\left\langle\alpha^{\vee}, \rho\right\rangle}^{0} d_{\rho+m \lambda+k \alpha} \log \left(k+\left\langle\alpha^{\vee}, \rho+m \lambda\right\rangle\right)
\end{aligned}
$$

using the skew-symmetry in the last equation. Notice that the last double sum is of order $O\left(m^{n} \log m\right)$. By approximating the first sum with integrals and doing partial integration we get

$$
-2 r(m)=\sum_{j} \int_{1}^{j m} \sum_{\alpha \in \Psi_{j}} d_{\rho+m \lambda-k \alpha} \log k d k+O\left(m^{n} \log m\right)
$$




$$
\begin{aligned}
= & \sum_{\alpha \in \Psi} \int_{1}^{\left\langle\alpha^{\vee}, m \lambda\right\rangle} d_{\rho+m \lambda-k \alpha} \log k d k+O\left(m^{n} \log m\right) \\
= & \left.\sum_{\alpha \in \Psi} \int_{0}^{x} d_{\rho+m \lambda-k \alpha} d k \log x\right|_{x=1} ^{\left\langle\alpha^{\vee}, m \lambda\right\rangle} \\
& -\sum_{\alpha \in \Psi} \int_{1}^{\left\langle\alpha^{\vee}, m \lambda\right\rangle} \int_{0}^{x} d_{\rho+m \lambda-k \alpha} d k \frac{d x}{x}+O\left(m^{n} \log m\right) \\
= & \sum_{\alpha \in \Psi} \int_{0}^{\left\langle\alpha^{\vee}, m \lambda\right\rangle} d_{\rho+m \lambda-k \alpha} d k \log \left\langle\alpha^{\vee}, m \lambda\right\rangle \\
& -\sum_{\alpha \in \Psi} \int_{0}^{\left\langle\alpha^{\vee}, m \lambda\right\rangle} \int_{0}^{x} d_{\rho+m \lambda-k \alpha} d k \frac{d x}{x}+O\left(m^{n} \log m\right)
\end{aligned}
$$

By the skew-symmetry of $d_{\rho+m \lambda-k \alpha}$, this equals

$$
\begin{aligned}
& \sum_{\alpha \in \Psi} \int_{0}^{\left\langle\alpha^{\vee}, \rho\right\rangle} d_{\rho+m \lambda-k \alpha} d k \log \left\langle\alpha^{\vee}, m \lambda\right\rangle \\
& -\sum_{\alpha \in \Psi} \int_{0}^{\left\langle\alpha^{\vee}, m \lambda\right\rangle} \int_{0}^{x} d_{\rho+m \lambda-k \alpha} d k \frac{d x}{x}+O\left(m^{n} \log m\right) \\
= & -\sum_{\alpha \in \Psi} \int_{0}^{\left\langle\alpha^{\vee}, m \lambda\right\rangle} \int_{0}^{x} d_{\rho+m \lambda-k \alpha} d k \frac{d x}{x}+O\left(m^{n} \log m\right)
\end{aligned}
$$

In particular, we see that $r(m)$ is of order $O\left(m^{n+1}\right)$, which can also be derived from equation (14). By decomposing into the $\Psi_{j}$-parts again and applying the Riemann-Roch theorem we get

$$
\begin{aligned}
2 r(m) & =\sum_{j} \int_{0}^{j m} \int_{0}^{x} \int_{M} \operatorname{Td}(T M) \operatorname{ch}\left(\psi^{-k} E_{j}\right) e^{m c_{1}\left(\mathcal{L}_{\lambda}\right)} d k \frac{d x}{x}+O\left(m^{n} \log m\right) \\
& =\int_{0}^{m} \int_{0}^{x} \int_{M} \operatorname{Td}(T M) \operatorname{ch}\left(\sum_{j} j \psi^{-j k} E_{j}\right) e^{m c_{1}\left(\mathcal{L}_{\lambda}\right)} d k \frac{d x}{x}+O\left(m^{n} \log m\right) \\
& =\int_{0}^{m} \int_{0}^{x} \int_{M} \operatorname{ch}\left(\sum_{j} j \psi^{-j k} E_{j}\right) e^{m c_{1}\left(\mathcal{L}_{\lambda}\right)} d k \frac{d x}{x}+O\left(m^{n} \log m\right)
\end{aligned}
$$

Hence we can express $r(m)$ as

$$
r(m)=\int_{M} \operatorname{Ht}\left(\sum_{j} j \psi^{j} E_{j}\right) e^{c_{1}\left(\mathcal{L}_{\lambda}\right)} \cdot m^{n+1}+O\left(m^{n} \log m\right) .
$$

This proves the above theorem. Q.E.D.

Remark. If $G_{c} / K$ is Hermitian symmetric a formula for $T\left(G_{c} / K, \bar{L}\right)$ is known [K2]. In this case we could have equally well worked with the difference $T_{g}\left(G_{c} / K, \bar{L}\right)-$ 
$T\left(G_{c} / K, \bar{L}\right)$ by arguing similarly as above, but avoiding the use of [BV]. In the case of Hermitian symmetric spaces there is a unique primitive $\lambda$. Using the classification of irreducible Hermitian symmetric spaces [He], one verifies that $\left\langle\alpha^{\vee}, \lambda\right\rangle$ equals either 1 or 2 for all $\alpha \in \Psi$.

Equation (15) provides a very effective way to compute the height. Namely, for $j \in \mathbf{N}$ consider the sum

$$
\begin{aligned}
f_{j}(m, k) & :=\sum_{\alpha \in \Psi_{j}} d_{\rho+m \lambda-k \alpha} \\
& =\sum_{\alpha \in \Psi_{j}} \prod_{\beta \in \Sigma_{K}^{+}}\left(1-k \frac{\left\langle\beta^{\vee}, \alpha\right\rangle}{\left\langle\beta^{\vee}, \rho\right\rangle}\right) \prod_{j^{\prime}} \prod_{\beta \in \Psi_{j^{\prime}}}\left(1+\frac{j^{\prime} m-k\left\langle\beta^{\vee}, \alpha\right\rangle}{\left\langle\beta^{\vee}, \rho\right\rangle}\right)
\end{aligned}
$$

and replace every power $k^{l}$ by $\frac{(m j)^{l+1}}{2(l+1)^{2}}$. The height is obtained by adding the coefficients of $m^{n+1}$ and multiplying with $(n+1)$ !. For $\alpha \in \Sigma$

$$
\#\left\{\beta \in \Sigma^{+} \mid\langle\alpha, \beta\rangle \neq 0\right\} \leq 2 c(G)-3
$$

where $c(G)$ is the Coxeter number of $G$ Bour, Ch. V $\S 6.1]$. Namely, by Bour, Ch. VI $\S 1$, Prop. 32], equality holds in (17) for root systems where all roots have the same length. Furthermore, for $B_{l}$ and $C_{l}$ the cardinality in (17) equals $2 c(G)-5$ and $c(G)-1$ (depending on the root), for $F_{4}$ it equals $2 c(G)-9=15$ and for $G_{2}$ it equals $c(G)-1=5$. Thus the degree in $k$ of $f_{j}(m, k)$ is less or equal to $2 c(G)-3$, and we find by using the same reformulation as in formula (13)

Corollary 8.2 There are $k_{\ell} \in \mathbf{Z}(1 \leq \ell \leq 2 c(G)-2)$ such that

$$
h\left(G / P, \overline{\mathcal{L}}_{\lambda}\right)=\sum_{\ell=1}^{2 c(G)-2} \frac{k_{\ell}}{2 \ell} .
$$

In other words, the largest power of a prime occurring in the denominator of $2 h\left(G / P, \overline{\mathcal{L}}_{\lambda}\right)$ is less or equal to $2 c(G)-2$.

Our computations of examples as well as Tamvakis' results in [T1], [T2], T3 strongly suggest the

Conjecture There are $k_{\ell} \in \mathbf{Z}(1 \leq \ell \leq c(G)-1)$ such that

$$
h\left(G / P, \overline{\mathcal{L}}_{\lambda}\right)=\sum_{\ell=1}^{c(G)-1} \frac{k_{\ell}}{2 \ell} .
$$

Also there is the following fixed point expression for $h\left(G / P, \overline{\mathcal{L}}_{\lambda}\right)$ : 
Lemma 8.3 Let $Y \in \mathfrak{g}$ act with isolated fixed points and let $\phi, \theta_{\nu}$ denote the angles of the action on $L_{\lambda \mid p}, T M_{\mid p}$ respectively for $p \in M^{Y}$. Then

$$
h\left(G / P, \overline{\mathcal{L}}_{\lambda}\right)=\sum_{p \in M^{Y}} \frac{1}{\prod_{\nu} \theta_{\nu}} \sum_{l=1}^{n+1} \sum_{j \in \mathbf{N}} \sum_{\theta_{\nu} \in \Psi_{j}} \frac{\phi^{n+1}-\phi^{n+1-l}\left(\phi-j \theta_{\nu}\right)^{l}}{2 l \theta_{\nu}} .
$$

Note that if $Y$ is an element of the Lie algebra of the maximal torus then there is a canonical isomorphism $M^{Y}=W_{G} / W_{K}$. The angle $\phi$ at $[w] \in W_{G} / W_{K}$ is given by $(2 \pi w \lambda)(Y)$ and the angle $\theta_{w \alpha}(\alpha \in \Psi)$ corresponding to $T M_{w \alpha}$ is given by $(2 \pi w \alpha)(Y)$. Thus, the formula in Lemma 8.3 reads

$$
\begin{aligned}
h\left(G / P, \overline{\mathcal{L}}_{\lambda}\right)= & \sum_{w \in W_{G} / W_{K}} \frac{1}{\prod_{\alpha \in \Psi} w \alpha(Y)} \\
& \cdot \sum_{l=1}^{n+1} \sum_{\alpha \in \Psi} \frac{(w \lambda(Y))^{n+1}-(w \lambda(Y))^{n+1-l}\left(S_{w \alpha} w \lambda(Y)\right)^{l}}{2 l w \alpha(Y)} .
\end{aligned}
$$

A good choice for $Y$ is the dual of $\rho$.

Proof: Applying the Bott residue formula to formula (13) yields

$$
h\left(G / P, \overline{\mathcal{L}}_{\lambda}\right)=\sum_{p \in M^{Y}} \sum_{l=0}^{n}\left(\begin{array}{c}
n+1 \\
l+1
\end{array}\right) \frac{\phi^{n-l}}{2(l+1) \prod \theta_{\nu}} \sum_{j \in \mathbf{N}} \sum_{\theta_{\nu} \in \Psi_{j}}\left(-\theta_{\nu}\right)^{l} j^{l+1}
$$

(alternatively, one could apply [K2, Th. 11] to $f_{j}(m, k)$ from equation 16] and proceed as in [KR3, proposition 3.7]). Using the formula

$$
\sum_{l=1}^{n}\left(\begin{array}{l}
n \\
l
\end{array}\right) \frac{x^{l}}{l}=\sum_{l=1}^{n} \frac{(1+x)^{l}-1}{l} \quad(x \in \mathbf{R}),
$$

we get the desired result. Q.E.D.

Example. We shall express the height of the Grassmannian $G(m, k)$ with $G(m, k)(\mathbf{C})=\mathbf{U}(m) / \mathbf{U}(k) \times \mathbf{U}(m-k)$ using Lemma 3.3. In this case, the Weyl group of $G$ is the permutation group $S_{m}$ of $m$ elements and the fixed point set can be identified with $S_{m} / S_{k} \times S_{m-k}$. Let $\left(\epsilon_{\nu}\right)_{\nu=1}^{m}$ be the cartesian base of $\mathbf{C}^{m}$, which we identify with $\mathfrak{t}^{\vee}$ as in [BtD, section V.6]. A short look to the classical tables of roots (e.g. Bour, VI, planche I] or BtD, Proposition V.6.2]) reveals that $\Psi=\left\{\epsilon_{\mu}-\epsilon_{\nu} \mid 1 \leq \mu \leq k<\nu \leq m\right\}$. Also, there is a unique positive primitive line bundle $\mathcal{L}_{\lambda}$ on $G(m, k)$ with $\lambda=\sum_{\nu=1}^{k} \epsilon_{k}$. In particular, $\Psi=\Psi_{1}$. Set $I_{m}:=\{1, \ldots, m\}$; when considering the action corresponding to $Y=\sum_{\nu=1}^{m} \nu \epsilon_{\nu}^{*}$, we get by Lemma 8.3

$$
h\left(G(m, k), \overline{\mathcal{L}}_{\lambda}\right)=\sum_{\substack{I \subseteq I_{m} \\ \# I=k}} \frac{\left(\sum_{c \in I} c\right)^{k(m-k)+1}}{\prod_{\substack{a \in I \\ b \in I_{m} \backslash I}}(a-b)} \sum_{\substack{a \in I \\ b \in I_{m} \backslash I}} \sum_{l=1}^{k(m-k)+1} \frac{1-\left(1-\frac{a-b}{\sum_{c \in I} c}\right)^{l}}{2 l(a-b)} .
$$


Example. Assume that $G / P$ is embedded as a hypersurface of degree $d$ into $\mathbf{P}^{n+1}$ via $\mathcal{L}_{\lambda}$. Let $N$ be its normal bundle. Classically, there is an exact sequence

$$
0 \rightarrow \mathcal{O} \rightarrow \bigoplus_{1}^{n+2} \mathcal{O}(1) \rightarrow T \mathbf{P}^{n+1} \rightarrow 0
$$

[Ha1, Ex. 8.20.1]. Furthermore, by the adjunction formula ([GH, p. 146])

$$
N=\mathcal{O}(d)_{\mid M} .
$$

Thus $\operatorname{ch}(T M)=\operatorname{ch}\left(T \mathbf{P}_{\mid M}^{n+1}\right)-\operatorname{ch}(N)=(n+2) e^{c_{1}(\mathcal{O}(1))}-1-e^{d c_{1}(\mathcal{O}(1))}$. Assume now that $E_{1}=T M$. Then equation (13) yields

$$
\begin{aligned}
h(G / P, \overline{\mathcal{O}(1)})= & \sum_{l=1}^{n} \frac{(-1)^{l}}{2(l+1)}\left(\begin{array}{c}
n+1 \\
l+1
\end{array}\right) \int_{M}\left(n+2-d^{l}\right) c_{1}(\mathcal{O}(1))^{n} \\
& +\frac{n+1}{2} \int_{M} n c_{1}(\mathcal{O}(1))^{n} \\
= & \sum_{l=1}^{n} \frac{d(-1)^{l}}{2(l+1)}\left(\begin{array}{c}
n+1 \\
l+1
\end{array}\right)\left(n+2-d^{l}\right)+\frac{n(n+1) d}{2} \\
= & \sum_{l=2}^{n+1} \frac{1}{2 l}\left[d(n+2)-1+(1-d)^{l}\right]
\end{aligned}
$$

By [Ko, Th. III.2.3 and next paragraph], all complex hypersurfaces of dimension $n>1$ with a non-trivial holomorphic $\mathbf{C}^{*}$-action have degree $d \leq 2$. We reobtain the formula for the height of $\mathbf{P}^{n}$ BoGS

$$
h\left(\mathbf{P}^{n}, \overline{\mathcal{O}(1)}\right)=\frac{n+1}{2} \sum_{k=1}^{n} \frac{1}{k}-\frac{n}{2} .
$$

Also [Bour. VI, planche IV] shows $\Psi=\Psi_{1}$ for the even-dimensional smooth quadric $Q_{2 m}$ with $Q_{2 m}(\mathbf{C})=\mathbf{S O}(2 m+2) / \mathbf{S O}(2 m) \mathbf{S O}(2)$. Thus we reobtain the result from $\mathrm{CM}$, Cor. 2.2.10]

$$
h\left(Q_{2 m}, \overline{\mathcal{O}(1)}\right)=(2 m+1) \sum_{k=1}^{2 m-1} \frac{1}{k}+\frac{1}{2} \sum_{k=1}^{m-1} \frac{1}{k}-2 m+1+\frac{1}{m} .
$$

Now consider the case of the odd-dimensional smooth quadric $Q_{2 m-1}$ with $Q_{2 m-1}(\mathbf{C})=\mathbf{S O}(2 m+1) / \mathbf{S O}(2 m-1) \mathbf{S O}(2)$. Identifying $\mathbf{C}^{m}$ with $\mathfrak{t}^{\vee}$ as in [BtD, proposition V.6.5] and denoting the cartesian base by $\left(\epsilon_{\nu}\right)_{\nu=1}^{m}$, we notice $\Psi=\left\{\epsilon_{1} \pm \epsilon_{\nu} \mid 1<\nu \leq m\right\} \cup\left\{\epsilon_{1}\right\}$ and $\lambda=\epsilon_{1}$ (compare also [Boun, VI,planche II]). Thus $T M=E_{1}+E_{2}$ with $E_{2}=\mathcal{O}(1)$. Hence there is an additional term to equation 18 given by

$$
\sum_{l=0}^{n} \frac{(-1)^{l}}{2(l+1)}\left(\begin{array}{c}
n+1 \\
l+1
\end{array}\right) \int_{M}\left(2^{l+1}-1\right) c_{1}(\mathcal{O}(1))^{n}
$$




$$
=\sum_{l=0}^{n} \frac{d(-1)^{l}}{2(l+1)}\left(\begin{array}{c}
n+1 \\
l+1
\end{array}\right)\left(2^{l+1}-1\right)=\sum_{l=1}^{n+1} \frac{(-1)^{l+1}}{l}
$$

and we find

$$
h\left(Q_{2 m-1}, \overline{\mathcal{O}(1)}\right)=(2 m+1) \sum_{k=1}^{2 m-1} \frac{1}{k}-\frac{1}{2} \sum_{k=1}^{m-1} \frac{1}{k}-2 m+1
$$

which is exactly the same value as obtained in $[\mathrm{CM}$, Cor. 2.2.10] for the singular quadric $X_{0}^{2}+\cdots+X_{2 m}^{2}=0$.

\section{References}

[Abbes] Abbes, A.: Hauteurs et discrétitude [d'après L. Szpiro, E. Ullmo et S. Zhang]. Sém. Bourbaki, no. 825 (1997).

[A1] Andersen, H. H.: Filtration of cohomology modules for Chevalley groups. Ann. Scient. Éc. Norm. Sup. 16, 495-528 (1983).

[A2] Andersen, H. H.: Torsion in the cohomology of line bundles on homogeneous spaces for Chevalley groups. Proc. Amer. Math. Soc. 96, 537-544 (1986).

[AB] Atiyah, M., Bott, R.: A Lefschetz fixed point formula for elliptic complexes II: Applications. Ann. of Math. 88, 451-491 (1968).

[BeGeV] Berline, N., Getzler, E., Vergne, M.: Heat kernels and Dirac operators: Springer 1992.

[Be] Besse, A.: Einstein manifolds: Erg. der Math. 10, Springer 1987.

[BV] Bismut, J.-M., Vasserot, E.:The asymptotics of the Ray-Singer analytic torsion associated with high powers of a positive line bundle. Comm. Math. Phys. 125, 355-367 (1989).

[BH] Borel, A., Hirzebruch, F.: Characteristic classes and homogeneous spaces, I. Am. J. Math. 80, 458-538 (1958).

[BoGS] Bost, J.-B., Gillet, H., Soulé, C.: Heights of projective varieties and positive Green forms. J. Amer. Math. Soc. 7, n. 4, 903-1027 (1994).

[Bott] Bott, R.: Homogeneous vector bundles. Ann. Math. 66, 203-248 (1957).

[Bour] Bourbaki, N.: Groupes et algèbres de Lie I-VIII: Hermann 1971-1975.

[BtD] Bröcker, T., tom Dieck, T.: Representations of Compact Lie Groups: GTM 98, Springer 1985. 
[CM] Cassaigne, J., Maillot, V.: Hauteurs des hypersurfaces et fonctions zêta d'Igusa. J. Number Theory 83, 226-255 (2000).

[Do] Donnelly, H.:Spectrum and the fixed point set of isometries I. Math. Ann. 224, 161-176 (1976).

[GH] Griffith, P., Harris, J.: Principles of algebraic geometry: Wiley Interscience 1978.

[GS5] Gillet, H., Soulé, C.: Analytic torsion and the arithmetic Todd genus. Topology 30, 21-54 (1991). With an Appendix by Zagier, D.

[Ha1] Hartshorne, R.: Algebraic geometry: Graduate texts in math. 52, Springer 1977.

[Ha2] Hartshorne, R.: Residues and duality: Springer Lecture Notes 20, Springer 1966.

[He] Helgason, S.: Differential geometry, Lie groups, and symmetric spaces: Academic Press 1978.

[Hu] Humphreys, J. E.: Introduction to Lie Algebras and Representation Theory: GTM 9, Springer 1972.

[J1] Jantzen, J. C.: Darstellungen halbeinfacher algebraischer Gruppen und zugeordnete kontravariant Formen. Bonner math. Schriften 67 (1973).

[J2] Jantzen, J. C.: Representations of Algebraic Groups: Academic Press 1987.

[Ko] Kobayashi, S.: Transformation Groups in Differential Geometry: Springer 1972.

[K1] Köhler, K.: Equivariant analytic torsion on $\mathbf{P}^{n}(\mathbf{C})$. Math. Ann. 297, 553-565 (1993).

[K2] Köhler, K.: Holomorphic torsion on Hermitian symmetric spaces. J. Reine Angew. Math. 460, 93-116 (1995).

[K3] Köhler, K.: Equivariant Reidemeister torsion on symmetric spaces. Math. Ann. 307, 57-69 (1997).

[KR1] Köhler, K., Roessler, D.: Un théorème du point fixe de Lefschetz en géométrie d'Arakelov. C. R. Acad. Sci. Paris 326, Série I, 719-722 (1998).

[KR2] Köhler, K., Roessler, D.: A fixed point formula of Lefschetz type in Arakelov geometry I: statement and proof. Preprint IHES/M/98/76 (1998). To appear in Inv. Math.

[KR3] Köhler, K., Roessler, D.: A fixed point formula of Lefschetz type in Arakelov geometry II: a residue formula. Preprint SFB 256 no. 604 (1999), Universität Bonn. To appear. 
[Ma1] Ma, Xiaonan: Formes de torsion analytique et familles de submersions I. Bull. Soc. Math. France 127, 541-621 (1999). Formes de torsion analytique et familles de submersions II. Asian J. Math. 4, 633-667 (2000).

[Ma2] Ma, Xiaonan: Submersions and equivariant Quillen metrics. Ann. Inst. Fourier (Grenoble) 50, 1539-1588 (2000).

[M] Maillot, V.: Un calcul de Schubert arithmétique. Duke Math. J. 80, 195221 (1995).

[N] Nielsen, N.: Theorie des Integrallogarithmus und verwandter Transcendenten: Teuber, Leipzig 1906.

[SGA3] Demazure, M., Grothendieck, A.: Schémas en Groupes, SGA3: Lecture Notes in Math. 151-153, Springer 1970.

[SB] Schmid, W., Bolton, V.: Discrete series. In: Bailey, T. N. (ed.) et al., Representation theory and automorphic forms. Providence, AMS. Proc. Symp. Pure Math. 61, 83-113 (1997).

[SABK] Soulé, C., Abramovich, D., Burnol, J.F. and Kramer, J.: Lectures on Arakelov Geometry (Cambridge studies in math. 33): Cambridge university press 1992 .

[T1] Tamvakis, H.: Schubert calculus on the arithmetic Grassmannian. Duke Math J. 98, 421-443 (1999).

[T2] Tamvakis, H.: Arithmetic intersection theory on flag varieties. Math. Ann. 314, 641-665 (1999).

[T3] Tamvakis, H.: Arakelov theory of the Lagrangian Grassmannian. J. Reine Angew. Math. 516, 207-223 (1999).

[V] Vogan, D.: Representation of real reductive Lie groups: Progress in Math. 15, Birkhäuser 1981.

[Z] Zhang, Shouwu: Positive line bundles on arithmetic varieties. J. Amer. Math. Soc. 8, 187-221 (1995). 